\title{
Bernstein and De Giorgi type problems: new results via a geometric approach
}

\author{
Alberto FARINA, BERARDINO SCIUNZI AND ENRICO VALDINOCI
}

\begin{abstract}
We use a Poincaré type formula and level set analysis to detect onedimensional symmetry of stable solutions of possibly degenerate or singular elliptic equations of the form

$$
\operatorname{div}(a(|\nabla u(x)|) \nabla u(x))+f(u(x))=0 .
$$

Our setting is very general and, as particular cases, we obtain new proofs of a conjecture of De Giorgi for phase transitions in $\mathbb{R}^{2}$ and $\mathbb{R}^{3}$ and of the Bernstein problem on the flatness of minimal area graphs in $\mathbb{R}^{3}$. A one-dimensional symmetry result in the half-space is also obtained as a byproduct of our analysis. Our approach is also flexible to very degenerate operators: as an application, we prove one-dimensional symmetry for 1-Laplacian type operators.
\end{abstract}

Mathematics Subject Classification (2000): 32H02 (primary); 30C45 (secondary).

\section{Introduction}

Of concern is a class of quasilinear (possibly singular or degenerate) elliptic equations in $\mathbb{R}^{N}$ with $N=2,3$. We prove one-dimensional symmetry of the solutions, thus showing that the important results of $[1-3,7,17]$ hold in a more general setting. The results we present are new even in the semilinear case, when the differential operator reduces to the standard Laplacian, since the nonlinearity we deal with may be less regular than what the previous literature requires.

The techniques used are novel and they are mainly based on a geometric formula, inspired by the work of [26,27], which bounds tangential gradients and curvatures of level sets of stable solutions in terms of suitable energy, or area, integrals.

As a by-product, we obtain a new result on mean curvature type operators which extends the Bernstein Theorem in [4] to a more general setting and to possibly non-homogeneous equations.

Work partially supported by MIUR Metodi variazionali ed equazioni differenziali nonlineari. Received January 31, 2008; accepted in revised form June 25, 2008. 
In further detail, the topic of this paper is the following. We consider weak solutions $u \in C^{1}\left(\mathbb{R}^{N}\right) \cap C^{2}(\{\nabla u \neq 0\})$ of equations of the form

$$
\operatorname{div}(a(|\nabla u(x)|) \nabla u(x))+f(u(x))=0 .
$$

We suppose that $f$ is a locally Lipschitz function and that $a \in C^{1}((0,+\infty))$ satisfies the following structural conditions:

$$
\begin{aligned}
a(t) & >0 \text { for any } t \in(0,+\infty), \\
a(t)+a^{\prime}(t) t & >0 \text { for any } t \in(0,+\infty) .
\end{aligned}
$$

The general form of (1.1) encompasses, as particular cases, many elliptic singular and degenerate operators such as the $p$-Laplacian, for $a(t)=t^{p-2}$, and the mean curvature, for $a(t)=1 / \sqrt{1+t^{2}}$.

We define $A: \mathbb{R}^{N} \backslash\{0\} \rightarrow \operatorname{Mat}(N \times N)$ by

$$
A_{h k}(\xi):=\frac{a^{\prime}(|\xi|)}{|\xi|} \xi_{h} \xi_{k}+a(|\xi|) \delta_{h k}
$$

for any $1 \leq h, k \leq N$.

We now introduce ${ }^{1}$ the following notation:

$$
\lambda_{1}(t):=a(t)+a^{\prime}(t) t, \quad \lambda_{2}(t)=\cdots=\lambda_{N}(t):=a(t),
$$

for any $t>0$.

It is also convenient to define

$$
\Lambda_{i}(t):=\int_{0}^{t} \lambda_{i}(|\tau|) \tau d \tau
$$

for $i=1,2$ and $t \in \mathbb{R}$ and

$$
F(t):=\int_{0}^{t} f(\tau) d \tau
$$

${ }^{1}$ In the literature, it is often assumed that $t a(t) \rightarrow 0^{+}$as $t \rightarrow 0^{+}$since in this case equation (1.1) is well-defined and many regularity results follow. In our case, this condition will be automatically fulfilled when condition (1.9), (B1) or (B2) are assumed. In such cases, the map $t \mapsto t a(t)$ is implicitly assumed to be extended at $t=0$ by continuity.

Analogously, when (B1) or (B2) are assumed, $A(\xi) \xi \rightarrow 0$ as $\xi \rightarrow 0$, hence the map $\xi \mapsto \xi A(\xi)$ is assumed to be extended at $\xi=0$ by continuity, even when $A(0)$ is not defined.

Note that $t a(t)>0$ for any $t>0$, due to (1.2), and the map $t \mapsto t a(t)$ is increasing, due to (1.3). As a consequence, $\operatorname{ta}(t) \in L_{\mathrm{loc}}^{\infty}([0,+\infty))$ and so $t^{2} a(t) \rightarrow 0^{+}$as $t \rightarrow 0^{+}$, and $\Lambda_{2}$ in (1.6) is well-defined.

On the other hand, in principle, $\Lambda_{1}$ in (1.6) may diverge. In our setting, conditions (A2), (B1) and (B2) will exclude this possibility. When condition (A1) is in force, we never use $\Lambda_{1}$. 
It is easily seen that (1.1) is equivalent to critical points of the formal variational principle

$$
\int \Lambda_{2}(|\nabla u|)-F(u) d x
$$

Theorem 1.1 below holds under very general assumptions on the differential operator. Namely, we will require in Theorem 1.1 that $a$ satisfies either (A1) or (A2), where:

(A1) $\{\nabla u=0\}=\emptyset$ and

$$
t^{2} \lambda_{1}(t) \in L_{\mathrm{loc}}^{\infty}([0,+\infty))
$$

(A2) We have that

$$
a \in C([0,+\infty))
$$

and

$$
\text { the map } t \mapsto t a(t) \text { belongs to } C^{1}([0,+\infty)) \text {. }
$$

In this case, we define $A_{h k}(0):=a(0) \delta_{h k}$.

It is customary to say (see, e.g., $[1,16,21])$ that a solution $u$ is stable if

$$
\int_{\mathbb{R}^{N}}(A(\nabla u(x)) \nabla \phi(x)) \cdot \nabla \phi(x)-f^{\prime}(u(x)) \phi^{2}(x) d x \geq 0
$$

for any smooth, compactly supported function $\phi: \mathbb{R}^{N} \rightarrow \mathbb{R}$, as long as ${ }^{2}$ the above integral is defined.

Of course, from the functional analysis point of view, the stability condition in (1.11) translates into the fact that the second variation of the functional in (1.7) is non-negative. Thence, in particular, such a condition is fulfilled by the minima of the functional.

We remark that in case $f$ is constant every solution is stable, since the matrix $A$ is positive definite (see Lemma 2.1 below).

In our very general framework, however, it is convenient to relax the definition of stability given in (1.11), since $f$ may not be everywhere differentiable.

2 We point out that, in general, the integral in (1.11) may be not well-defined for some test functions in case the gradient of $u$ vanishes, since $A$ may diverge there. In our setting, however, such integral will be always well-defined, due to (A1) and (A2).

We also note that, under condition (A2), $A \in C\left(\mathbb{R}^{N}\right)$, since, for any $t>0$,

$$
a(t)-\frac{1}{t} \int_{0}^{t}(\tau a(\tau))^{\prime} d \tau=0
$$

and so

$$
0=a(0)-\lim _{t \rightarrow 0^{+}}(t a(t))^{\prime}=\lim _{t \rightarrow 0^{+}} t a^{\prime}(t) .
$$

Conditions (A1) and (A2) are indeed quite different in spirit, as the example in Proposition 7.2 below will also show. 
For this, we consider the sets

$$
\mathcal{G}:=\left\{t \in \mathbb{R} \text { such that } f^{\prime}(t) \text { exists }\right\}
$$

and

$$
\mathcal{N}:=\mathbb{R} \backslash \mathcal{G}
$$

We recall that

$\mathcal{N}$ is Borel (see [10, page 82])

and with zero Lebesgue measure (see [10, page 81$]$ ).

We consider the sets

$$
\mathcal{N}_{u}:=u^{-1}(\mathcal{N})=\left\{x \in \mathbb{R}^{N} \text { such that } u(x) \in \mathcal{N}\right\}
$$

and

$$
\mathcal{G}_{u}:=\mathbb{R}^{N} \backslash \mathcal{N}_{u} .
$$

We thus replace the definition of stability in (1.11) with

$$
\int_{\mathbb{R}^{N}}(A(\nabla u(x)) \nabla \phi(x)) \cdot \nabla \phi(x)-\int_{\mathcal{G}_{u}} f^{\prime}(u(x)) \phi^{2}(x) d x \geq 0 .
$$

Notice that (1.13) makes sense ${ }^{3}$ when $f$ is just locally Lipschitz and it agrees with (1.11) when $f$ admits derivatives everywhere.

Then, our symmetry result in $\mathbb{R}^{2}$ is the following:

Theorem 1.1. Let $N=2$. Let $u \in C^{1}\left(\mathbb{R}^{2}\right) \cap C^{2}(\{\nabla u \neq 0\})$, with $\nabla u \in L^{\infty}\left(\mathbb{R}^{2}\right) \cap$ $W_{\mathrm{loc}}^{1,2}\left(\mathbb{R}^{2}\right)$, a and $f$ be as in (1.1), (1.2) and (1.3).

Assume that either (A1) or (A2) holds.

Suppose that $u$ is stable.

Then, $u$ has one-dimensional symmetry, in the sense that there exists $\bar{u}: \mathbb{R} \rightarrow$ $\mathbb{R}$ and $\omega \in S^{1}$ in such a way that $u(x)=\bar{u}(\omega \cdot x)$, for any $x \in \mathbb{R}^{2}$.

We observe that, as paradigmatic examples satisfying the assumptions of Theorem 1.1, one may take the $p$-Laplacian, with any $p \in(1,+\infty)$ if $\{\nabla u=0\}=\emptyset$ and any $p \in[2,+\infty)$ if $\{\nabla u=0\} \neq \emptyset$, or the mean curvature operator.

3 Another way to make sense of (1.11) when $f$ does not admit derivatives everywhere is to ask that (1.11) holds for any $\phi:=|\nabla u| \varphi$, for any test function $\varphi$.

This is a good definition, since the map $x \mapsto f(u(x))$ is locally Lipschitz and so

$$
\nabla(f(u)) \cdot \nabla u \varphi^{2}=f^{\prime}(u)|\nabla u|^{2} \varphi^{2}=f^{\prime}(u) \phi^{2}
$$

almost everywhere, giving sense to the last integrand in (1.11).

The only use of test functions of the type $|\nabla u| \varphi$ in (1.11) would be, in fact, enough for our purposes: see the choice performed on page 754 below. 
We also remark that the assumption that $\nabla u \in W_{\text {loc }}^{1,2}\left(\mathbb{R}^{N}\right)$ is always verified in the $p$-Laplacian case if either $\{\nabla u=0\}=\emptyset$ or if $1<p<3$ (see, e.g. (2.2.2) in [28] for the case $1<p \leq 2$, and [6, Theorem 1.1 and Proposition 2.2] for the case $2 \leq p<3$ ).

As is well-known, the assumption that $u \in C^{1}\left(\mathbb{R}^{N}\right) \cap C^{2}(\{\nabla u \neq 0\})$ is also fulfilled in the $p$-Laplacian case (see, e.g. [9, 28, 29]).

The assumption that $|\nabla u|$ is bounded cannot be removed, as we will show by explicit counterexamples in Proposition 3.1.

In the following Theorem 1.2, some further structural assumptions on $a$ will be required. These assumptions, which will use the notation introduced here above, are still quite general, and make it possible to use some results of [5,7].

Following [5,7], we list these further assumptions by asking that $a$ satisfies either (B1) or (B2), where:

(B1) There exist $p>1, \epsilon \geq 0$, and $c^{\star} \in(0,1)$ such that for every $\xi, \zeta \in \mathbb{R}^{N} \backslash\{0\}$, one has:

$$
c^{\star}(\epsilon+|\xi|)^{p-2}|\zeta|^{2} \leq A_{h k}(\xi) \zeta_{h} \zeta_{k} \leq \frac{1}{c^{\star}}(\epsilon+|\xi|)^{p-2}|\zeta|^{2}
$$

(B2) There exist $c^{\star} \in(0,1)$ such that for every $\xi, \zeta \in \mathbb{R}^{N} \backslash\{0\}$, one has:

$$
\begin{aligned}
\frac{c^{\star}}{(1+|\xi|)} & \leq a(|\xi|) \leq \frac{1}{c^{\star}(1+|\xi|)} \\
c^{\star} \frac{|\zeta|^{2}+(\zeta \cdot \xi)^{2}}{1+|\xi|} & \leq A_{h k}(\xi) \zeta_{h} \zeta_{k} \leq \frac{1}{c^{\star}} \frac{|\zeta|^{2}+(\zeta \cdot \xi)^{2}}{1+|\xi|} .
\end{aligned}
$$

Conditions (B1) and (B2) are fulfilled, for instance, by $p$-Laplacian type and mean curvature type ${ }^{4}$ operators (see, e.g. (1.4) and (1.5) of [5] or (2.12) and (2.13) of [7]).

In this framework, the following result holds:

Theorem 1.2. Let $N=3, u \in C^{1}\left(\mathbb{R}^{3}\right) \cap W^{1, \infty}\left(\mathbb{R}^{3}\right)$, and a and $f$ as in (1.1). Assume that either (B1) or (B2) holds. Suppose also that

$$
a \in C_{\mathrm{loc}}^{1,1}(0,+\infty)
$$

and that $\partial_{x_{3}} u>0$.

Then, $u$ has one-dimensional symmetry, in the sense that there exists $\bar{u}: \mathbb{R} \rightarrow$ $\mathbb{R}$ and $\omega \in S^{2}$ in such a way that $u(x)=\bar{u}(\omega \cdot x)$, for any $x \in \mathbb{R}^{3}$.

4 We kept the distinction between (B1) and (B2) mainly to be uniform with the existing literature. In fact, in our setting, condition (B2) can be seen as a particular case of (B1) with $p=2$, because in Theorem 1.2 all the considered solutions have bounded gradient. 
We remark that we will prove a stronger version of Theorem 1.2. Namely, we prove that Theorem 1.2 holds by replacing the assumption $\partial_{x_{3}} u>0$ with the following, a priori ${ }^{5}$ weaker, ones: $\partial_{x_{3}} u \geq 0,\{\nabla u=0\}=\emptyset$ and $u$ stable.

Let us make some comments on the above assumptions. First of all, the hypotheses of Theorem 1.2 are obviously stronger than the ones of Theorem 1.1 and, in particular, (B1) or (B2) imply (1.2) and (1.3). Moreover, (B1) or (1.15) bound the eigenvalues of $A(\xi)$ from above by $(\epsilon+|\xi|)^{p-2}$ and $1+|\xi|^{2}$, respectively. Since $\lambda_{1}$ is an eigenvalue (see Lemma 2.1 below), we also have that (B1) or (B2) imply (1.8).

Furthermore, the stability condition in (1.13) is implied by the assumption $\partial_{x_{N}} u>0$ (this is a standard fact for phase transitions and details fitting our setting are given in Lemma 7.1 below).

We also note that (B1) or (B2) give a regularity theory for $u$, namely $u \in$ $C_{\text {loc }}^{1, \alpha}\left(\mathbb{R}^{3}\right)$ and so, since $\{\nabla u=0\}=\emptyset$, we have that $u \in C^{2}\left(\mathbb{R}^{3}\right)$ (see footnote 4 here and [7, page 459] and references therein).

We remark again that paradigmatic examples satisfying the hypotheses of Theorem 1.2 are the $p$-Laplacian operator, for any $p \in(1,+\infty)$, and the mean curvature operator.

Theorems 1.1 and 1.2 reduce, in the particular case of $a(t)=1$ and $f(t)=$ $t-t^{3}$, to a famous problem posed by De Giorgi on [8, page 175].

In this respect, Theorems 1.1 and 1.2 are extensions of the celebrated results in $[3,17]$ for $N=2$ and $[1,2]$ for $N=3$ to more general, possibly degenerate, operators and to less regular nonlinearities - to the best of our knowledge, for instance, $f$ is needed to be at least $C^{1}$ in the literature, and such condition is crucial in $[1,2]$ to pass to the limit in the linearized equation and in the stability condition.

One-dimensional symmetry for solutions of non-uniformly elliptic operators has also been dealt with in $[5,7,12,30]$. Theorems 1.1 and 1.2 strengthen these results: for instance, the monotonicity assumption in [7, Theorem 7.1] is weakened in favor of the stability assumption of Theorem 1.1 here, and no additional limit requirement is needed in our Theorem 1.2, in contrast with [7, Theorem 8.1] and [30, Theorem 1.4] (on the other hand, the latter result holds for $N \leq 8$ ).

Results and techniques developed here are also the keystone of the forthcoming papers [15] and [25], in which higher dimensional problems and fractional operators will be considered.

We remark that Theorems 1.1 and 1.2 are somehow bias, in the sense that the proof of Theorem 1.2 does not rely on Theorem 1.1 but directly on the geometric level sets estimates, because some of the hypotheses of Theorem 1.2, such as the strict monotonicity and the stability conditions, would be lost in the limit (that is, limits of strictly monotone functions may be not stricly monotone, and (1.13) does not pass to the limit if $f^{\prime}$ is not continuous).

5 A posteriori, Theorem 1.2 under the weaker assumptions implies that the solution is onedimensional, thence, since $\{\nabla u=0\}=\emptyset$, we have that $u$ is strictly monotone in some direction $\omega$, which is not necessarily $e_{3}$. 
As a consequence of Theorem 1.2, we obtain the following result about symmetry in the half space.

Corollary 1.3. Let $g$ be locally Lipschitz continuous in $[0,+\infty)$, with $g(0)=0$. Let $u \in C^{2}\left(\mathbb{R}^{2} \times[0,+\infty)\right) \cap L^{\infty}\left(\mathbb{R}^{2} \times[0,+\infty)\right)$ be such that

$$
\begin{aligned}
& \Delta u(x)+g(u(x))=0 \\
& u(x)>\text { for any } x \in \mathbb{R}^{2} \times(0,+\infty), \\
& \text { and } u\left(x^{\prime}, 0\right)=0 \text { for any } x^{\prime} \in \mathbb{R}^{2} .
\end{aligned}
$$

Then, $u$ has one-dimensional symmetry, in the sense that there exists $\bar{u}: \mathbb{R} \rightarrow \mathbb{R}$ in such a way that $u\left(x^{\prime}, x_{3}\right)=\bar{u}\left(x_{3}\right)$, for any $\left(x^{\prime}, x_{3}\right) \in \mathbb{R}^{2} \times[0,+\infty)$.

Corollary 1.3 is an extension of [3, Theorem 1.5]. In fact, the results of Corollary 1.3 here and of [3, Theorem 1.5] hold under different hypotheses: Theorem 1.5 for $N=3$ in [3] is valid assuming only that $g(0) \geq 0$, but requiring $g$ to be $C^{1}$, while here we need $g(0)=0$ but we may allow less regularity (once more, the condition that $g^{\prime}$ is continuous was not merely technical in [3], since it was needed for taking limits: see, in particular, [3, Lemma 3.1 on page 84$]$ ).

We now state a Bernstein type result:

Theorem 1.4. Let $N=2$. Let $u \in C^{2}\left(\mathbb{R}^{2}\right)$, $a$ and $f$ be as in (1.1), (1.2) and (1.3). Suppose also that $f$ has a sign (meaning that either $f \geq 0$ or $f \leq 0$ ), that

$$
|a(t) t| \leq C
$$

for any $t \geq C$, and that

$$
t^{2} \lambda_{1}(t) \leq C a(t)
$$

for any $t \geq C$, for a suitable $C \geq 1$.

Suppose that either (A1) or (A2) holds, and that $u$ is stable.

Then, $u$ possesses one-dimensional symmetry.

As a particular case, one may take $f:=0$ and $a(t):=1 / \sqrt{1+t^{2}}$ in Theorem 1.4: in such a case, our result reduces to the very famous fact that minimal surfaces which are graphs in $\mathbb{R}^{3}$ are one-dimensional and, as a consequence, affine (see [4]): this classical result is therefore extended here to more general types of equations, thanks to our different approach.

We recall also that, in the minimal surface case, where $f:=0$ and $a(t):=$ $1 / \sqrt{1+t^{2}}$, any solution of (1.1) is automatically stable, since $f^{\prime}$ vanishes identically.

The main novelty with respect to the techniques already available in the literature for dealing with one-dimensional symmetries is here the use of a Poincaré type formula (given in Theorem 2.5), which extends a similar one of [26,27]. Such a formula is geometric in spirit, since it involves the curvature and the tangential gradients of level sets. 
In some sense, this approach makes it possible to deal with the problems of Bernstein and De Giorgi, which have a geometric flavor, in a more geometric way. Such a geometric answer to problems that are of geometric nature has also some technical advantages. For instance, it will give us the possibility of proving Theorems 1.1 and 1.2 without any assumptions at infinity (which are needed in $[7,22,30])$. It also allows us to deal with a less regular $f$ with respect to $[1,2]$ and, differently from [22,30], no growth assumptions from the minima of $F$ are needed here.

This generality is accomplished thanks to the geometry encoded in the Poincare type formula of Theorem 2.5: indeed, while it is not possible to "push" the stability in (1.13) towards infinity (unless $f^{\prime}$ is continuous), the information on the level sets will remain at infinity.

In this sense, Theorem 2.5 will allow us to obtain first some geometric information and then to push it to infinity.

We also mention that, differently from $[2,22,30]$, we do not need here to assume the existence of a monotone one-dimensional profile. The monotone onedimensional profile was important in [2] for bounding the energy and in [22,30] to build suitable barriers, which are not needed in our construction. In fact, our limiting profile may have somewhat wild behaviors (see Lemma 4.14 and the examples in Section 7.2).

For a survey of geometric problems related to the one considered here, in connection with Liouville type results, see also [13].

We finish with an application to very degenerate operators of 1-Laplacian type.

For this, we consider the following weakened version of (1.3):

$$
a(t)+a^{\prime}(t) t \geq 0 \text { for any } t \in(0,+\infty) .
$$

We prove that

Theorem 1.5. Suppose that $f$ is locally Lipschitz, that $a \in C^{1}((0,+\infty))$ satisfies (1.2), (1.19) and that (A1) holds.

Let $u \in C^{2}\left(\mathbb{R}^{2}\right)$ be a stable solution of $(1.1)$ in $\mathbb{R}^{2}$, with $|\nabla u| \in L^{\infty}\left(\mathbb{R}^{2}\right)$.

Then, $u$ has one-dimensional symmetry.

The easiest case of operators fulfilling the assumptions of Theorem 1.5 is given by $a(t)=1 / t$ for all (or for some) $t>0$, that are 1-Laplacian type operators. We remark that the assumption that $\nabla u$ does not vanish, which is contained in (A1), is needed to make sense of (1.1).

The organization of the paper is the following. In Section 2, we prove Theorem 1.1. Section 3 contains examples showing the optimality of the assumptions of Theorem 1.1. We then provide two proofs of Theorem 1.2. Namely, Section 4 contains some general preliminaries, needed for both the proofs of Theorem 1.2, Section 5 gives the details of the first proof, based on a capacity argument, and Section 6 presents the second proof, which exploits a Liouville type result (the end of the latter proof is closely related to similar arguments in $[1,2,7])$. 
In the semilinear model, monotone solutions are known to be stable: modifications of standard arguments are given in Section 7 to show that the same holds in our setting.

The proof of Corollary 1.3 and Theorem 1.4 are then given in Sections 8 and 9, respectively.

Theorem 1.5 is proven in Section 10.

\section{Proof of Theorem 1.1}

We borrow a large number of ideas from [11] and exploit some techniques of [26, 27].

\subsection{Algebraic manipulations}

In the sequel, we implicitly assume $N \geq 2$.

Lemma 2.1. For any $\xi \in \mathbb{R}^{N} \backslash\{0\}$, the matrix $A(\xi)$ is symmetric and positive definite and its eigenvalues are $\lambda_{1}(|\xi|), \ldots, \lambda_{N}(|\xi|)$.

Moreover,

$$
A(\xi) \xi \cdot \xi=|\xi|^{2} \lambda_{1}(|\xi|)
$$

Proof. The formula in (2.1) is straightforward.

Fixed $\xi \in \mathbb{R}^{N} \backslash\{0\}$, the matrix $A(\xi)$ is obviously symmetric. What is more, $A(\xi) \xi=\lambda_{1}(|\xi|) \xi$ and $A(\xi) v=\lambda_{2}(|\xi|) v$ for any $v \in \mathbb{R}^{N}$ such that $v \cdot \xi=0$. This says that the eigenvalues of $A(\xi)$ are as in (1.5). Such eigenvalues are positive, due to (1.2) and (1.3), and so $A(\xi)$ is positive definite.

Consequences of Lemma 2.1 are that

$$
\Lambda_{i}(-t)=\Lambda_{i}(t)>0
$$

for any $t \in \mathbb{R} \backslash\{0\}$, and that

$$
0 \leq A(\xi)(V-W) \cdot(V-W)=A(\xi) V \cdot V+A(\xi) W \cdot W-2 A V \cdot W,
$$

for any $V, W \in \mathbb{R}^{N}$ and any $\xi \in \mathbb{R}^{N} \backslash\{0\}$.

Lemma 2.2. Let $u \in C^{1}\left(\mathbb{R}^{N}\right) \cap C^{2}(\{\nabla u \neq 0\})$, with $\nabla u \in W_{\mathrm{loc}}^{1,2}\left(\mathbb{R}^{N}\right)$ be a weak solution of (1.1). Suppose that either (A2) holds or that $\{\nabla u=0\}=\emptyset$.

Then, $u_{j}$ is a weak solution of

$$
\operatorname{div}\left(A(\nabla u(x)) \nabla u_{j}(x)\right)+f^{\prime}(u(x)) u_{j}(x)=0
$$

for any $j=1, \ldots, N$. 
Proof. First of all, we observe that

the map $x \mapsto W(x):=a(|\nabla u(x)|) \nabla u(x)$ belongs to $W_{\text {loc }}^{1,1}\left(\mathbb{R}^{N}, \mathbb{R}^{N}\right)$.

Indeed, this is obvious if $\{\nabla u=0\}=\emptyset$, while, if (A2) holds, we see that the map

$$
\mathbb{R}^{N} \ni \xi \mapsto \Psi(\xi):=a(|\xi|) \xi
$$

belongs to $W_{\text {loc }}^{1, \infty}\left(\mathbb{R}^{N}, \mathbb{R}^{N}\right)$, and so (2.4) follows by writing $W(x)=\Psi(\nabla u(x))$.

From (2.4), we have that

$$
-\int_{\mathbb{R}^{N}} \partial_{j}(a(|\nabla u|) \nabla u) \cdot \psi d x=\int_{\mathbb{R}^{N}} a(|\nabla u|) \nabla u \cdot \partial_{j} \psi d x,
$$

for any $\psi \in C_{0}^{\infty}\left(\mathbb{R}^{N}, \mathbb{R}^{N}\right)$.

Also, by (2.4) and Stampacchia's theorem (see, for instance, [19, Theorem 6.19]), we get that $\partial_{j} W(x)=0$ for almost any $x \in\{W=0\}$, that is

$$
\partial_{j}(a(|\nabla u(x)|) \nabla u(x))=0
$$

for almost any $x \in\{\nabla u=0\}$.

Analogously, making use again of Stampacchia's theorem and (A2), we see that $\nabla u_{j}(x)=0$, and so $A(\nabla u(x)) \nabla u_{j}(x)=0$, for almost any $x \in\{\nabla u=0\}$.

A direct computation also shows that

$$
\partial_{j}(a(|\nabla u|) \nabla u)=A(\nabla u) \nabla u_{j}
$$

on $\{\nabla u \neq 0\}$.

As a consequence,

$$
\partial_{j}(a(|\nabla u|) \nabla u)=A(\nabla u) \nabla u_{j}
$$

almost everywhere.

Let now $\phi \in C_{0}^{\infty}\left(\mathbb{R}^{N}\right)$. We use the above observations to obtain that

$$
\begin{aligned}
& -\int_{\mathbb{R}^{N}} A(\nabla u) \nabla u_{j} \cdot \nabla \phi+f^{\prime}(u) u_{j} \phi d x \\
= & -\int_{\mathbb{R}^{N}} \partial_{j}(a(|\nabla u|) \nabla u) \cdot \nabla \phi+\partial_{j}(f(u)) \phi d x \\
= & \int_{\mathbb{R}^{N}} a(|\nabla u|) \nabla u \cdot \nabla \phi_{j}+f(u) \phi_{j} d x,
\end{aligned}
$$

which vanishes due to (1.1). 
The careful reader may easily convince herself or himself that the proof of Lemma 2.2 holds, in fact, assuming $\nabla u \in W_{\text {loc }}^{1,1}\left(\mathbb{R}^{N}\right)$ : since such a generality is not needed here, we assumed, for simplicity, $\nabla u \in W_{\text {loc }}^{1,2}\left(\mathbb{R}^{N}\right)$ in order to use the above result in Lemma 2.4.

We will now consider the tangential gradient with respect to a regular level set. That is given $v \in C^{1}\left(\mathbb{R}^{N}\right)$, we define the level set of $v$ at $x$ as

$$
L_{v, x}:=\left\{y \in \mathbb{R}^{N} \text { such that } v(y)=v(x)\right\} .
$$

If $\nabla v(x) \neq 0, L_{v, x}$ is a hypersurface near $x$ and one can consider the projection of any vector onto the tangent plane: in particular, the tangential gradient, which will be denoted as $\nabla_{L_{v, x}}$, is the projection of the gradient. That is, given $f \in C^{1}\left(B_{\rho}(x)\right)$, for $\rho>0$, we set

$$
\nabla_{L_{v, x}} f(x):=\nabla f(x)-\left(\nabla f(x) \cdot \frac{\nabla v(x)}{|\nabla v(x)|}\right) \frac{\nabla v(x)}{|\nabla v(x)|} .
$$

Lemma 2.3. Let $U \subseteq \mathbb{R}^{N}$ be an open set, $v \in C^{2}(U)$ and $x \in U$ be such that $\nabla v(x) \neq 0$. Then,

$$
\begin{gathered}
a(|\nabla v(x)|)\left[|\nabla| \nabla v|(x)|^{2}-\sum_{j=1}^{N}\left|\nabla v_{j}(x)\right|^{2}\right]-a^{\prime}(|\nabla v(x)|)|\nabla v(x)|\left|\nabla_{L_{v, x}}\right| \nabla v|(x)|^{2} \\
=(A(\nabla v(x))(\nabla|\nabla v|(x))) \cdot(\nabla|\nabla v|(x))-\left(A(\nabla v(x)) \nabla v_{j}(x)\right) \cdot \nabla v_{j}(x) .
\end{gathered}
$$

Proof. We use $\nabla_{L}, a$ and $A$ as a short-hand notation for $\nabla_{L_{v, x}}, a(|\nabla v(x)|)$ and $A(\nabla v(x))$ respectively. We observe that, from (2.6),

$$
\left|\nabla_{L_{v, x}} f\right|^{2}=|\nabla f|^{2}-\left(\nabla f \cdot \frac{\nabla v}{|\nabla v|}\right)^{2}
$$

for any smooth function $f$.

It is also straightforward that

$$
\partial_{j}|\nabla f|=\frac{\nabla f \cdot \nabla f_{j}}{|\nabla f|}
$$

at points where $\nabla f \neq 0$. 
We exploit these observations and (1.4) to make the following computation:

$$
\begin{aligned}
( & A(\nabla|\nabla v|)) \cdot(\nabla(|\nabla v|))-\left(A \nabla v_{j}\right) \cdot \nabla v_{j} \\
& -\left.a|\nabla| \nabla v\right|^{2}+a\left|\nabla v_{j}\right|\left|\nabla v_{j}\right|+a^{\prime}|\nabla v|\left|\nabla_{L}\right| \nabla v \|^{2} \\
= & \frac{a^{\prime}}{|\nabla v|} v_{h} v_{k} \partial_{h}|\nabla v| \partial_{k}|\nabla v| \\
& -\frac{a^{\prime}}{|\nabla v|} v_{h} v_{k} v_{j h} v_{j k}+a^{\prime}|\nabla v|\left[|\nabla| \nabla v \|^{2}-\left(\nabla|\nabla v| \cdot \frac{\nabla v}{|\nabla v|}\right)^{2}\right] \\
= & -\frac{a^{\prime}}{|\nabla v|}\left(\nabla v \cdot \nabla v_{j}\right)\left(\nabla v \cdot \nabla v_{j}\right)+a^{\prime}|\nabla v||\nabla| \nabla v \|^{2} \\
= & 0,
\end{aligned}
$$

where the index summation convention is understood.

\subsection{An elementary density argument}

In Theorem 2.5 below, we will need to use the stability condition (1.13) for a less regular test function. To this extent, we need the following observation:

Lemma 2.4. Suppose that either (A2) holds or that $\{\nabla u=0\}=\emptyset$. Let $u \in$ $C^{1}\left(\mathbb{R}^{N}\right)$ be a stable weak solution of (1.1). Then, (1.13) holds for any $\phi \in W_{0}^{1,2}(B)$ and any ball $B \subset \mathbb{R}^{N}$.

Also, under the assumptions of Lemma 2.2,

$$
\int_{\mathbb{R}^{N}} A(\nabla u) \nabla u_{j} \cdot \nabla \phi-f^{\prime}(u) u_{j} \phi d x=0
$$

for any $j=1, \ldots, N$, any $\phi \in W_{0}^{1,2}(B)$ and any ball $B \subset \mathbb{R}^{N}$.

We omit the standard proof of such result (for full details see [14]).

\subsection{Extension of a Poincaré type formula by Sternberg and Zumbrun}

Given $y \in L_{u, x} \cap\{\nabla u \neq 0\}$, let $\kappa_{1, u}(y), \ldots, \kappa_{(N-1), u}(y)$ denote the principal curvatures of $L_{u, x}$ at $y$. In the sequel, we will often use $\kappa_{\ell}$ as a short-hand notation for $\kappa_{\ell, u}$, when no ambiguity is possible.

Tangential gradients and curvatures may be conveniently related: indeed, according to formula (2.1) of [26],

$$
\sum_{j}\left|\nabla v_{j}\right|^{2}-\left|\nabla_{L}\right| \nabla v||^{2}-|\nabla| \nabla v||^{2}=|\nabla v|^{2} \sum_{\ell} \kappa_{\ell, v}^{2},
$$

on $\{\nabla v \neq 0\}$, for any $v \in C^{2}(\{\nabla v \neq 0\})$. 
In the subsequent Theorem 2.5, we give an extension of a formula obtained in $[26,27]$. This formula relates the stability of the equation with the principal curvatures of the corresponding level set and with the tangential gradient of the solution. Since this formula bounds a weighted $L^{2}$-norm of any test function by a weighted $L^{2}$-norm of its gradient, we may see this formula as a weighted Poincaré type inequality.

The proof we present is a variation of the one given in [26,27]: the main difference is that we exploit here the results of Section 2.1 in order to go back to the usual notion of curvatures and tangents instead of the one in the metric structure induced by $A$.

Theorem 2.5. Let $\Omega \subseteq \mathbb{R}^{N}$ be open (not necessarily bounded). Let $u \in C^{1}(\Omega) \cap$ $C^{2}(\Omega \cap\{\nabla u \neq 0\})$ with $\nabla u \in W_{\text {loc }}^{1,2}(\Omega)$ be a stable weak solution of (1.1) in $\Omega$. Suppose that either (A2) holds or that $\{\nabla u=0\}=\emptyset$.

For any $x \in \Omega \cap\{\nabla u \neq 0\}$ let $L_{u, x}$ denote the level set of $u$ at $x$, according to (2.5).

Let also $\lambda_{1}(|\xi|)$ and $\lambda_{2}(|\xi|)$ be as in (1.5).

Then,

$$
\begin{aligned}
& \int_{\Omega \cap\{\nabla u \neq 0\}} {\left[\lambda_{1}(|\nabla u(x)|)\left|\nabla_{L_{u, x}}\right| \nabla u|(x)|^{2}\right.} \\
&\left.+\lambda_{2}(|\nabla u(x)|)|\nabla u(x)|^{2} \sum_{\ell=1}^{N-1} \kappa_{\ell}^{2}(x)\right] \varphi^{2}(x) d x \\
& \leq \int_{\Omega}|\nabla u(x)|^{2}(A(\nabla u(x)) \nabla \varphi(x)) \cdot \nabla \varphi(x) d x
\end{aligned}
$$

for any locally Lipschitz, function $\varphi: \Omega \rightarrow \mathbb{R}$ whose support is compact and contained in $\Omega$.

Proof. We make use of $\lambda_{i}, \kappa_{i}, \nabla_{L}, a$ and $A$ as a short-hand notation for $\lambda_{i}(|\nabla u(x)|)$, $\kappa_{i}(x), \nabla_{L_{u, x}}, a(|\nabla u(x)|)$ and $A(\nabla u(x))$ respectively.

Since the maps $x \mapsto u_{j}(x)$ and $x \mapsto|\nabla u(x)|$ belong to $W_{\text {loc }}^{1,2}(\Omega)$, Stampacchia's theorem (see, e.g., [19, Theorem 6.19]) yields that

$$
\nabla|\nabla u|=0 \text { almost everywhere on }\{|\nabla u|=0\}
$$

and

$$
\nabla u_{j}=0 \text { almost everywhere on }\{|\nabla u|=0\} \subseteq\left\{u_{j}=0\right\},
$$

for any $j=1, \ldots, N$.

We take now $\phi:=u_{j} \varphi^{2}$ in (2.8) and we sum over $j$ to see that

$$
\int\left(A \nabla u_{j}\right) \cdot \nabla\left(u_{j} \varphi^{2}\right)-f^{\prime}(u)|\nabla u|^{2} \varphi^{2} d x=0 .
$$


Notice also that (1.12) and of [19, Theorem 6.19] give that

$$
\nabla u=0 \text { almost everywhere on } \mathcal{N}_{u}
$$

and therefore

$$
\int_{\mathcal{G}_{u}} f^{\prime}(u)|\nabla u|^{2} \varphi^{2}=\int_{\Omega} f^{\prime}(u)|\nabla u|^{2} \varphi^{2} .
$$

We thus exploit (1.13) with $\phi:=|\nabla u| \varphi$. Note that this choice is possible, thanks to Lemma 2.4. The use of (2.11) and Lemma 2.3 then implies

$$
\begin{aligned}
0 \leq & \int|\nabla u|^{2}(A \nabla \varphi) \cdot \nabla \varphi+\varphi^{2}(A \nabla|\nabla u|) \cdot \nabla|\nabla u| \\
& +2 \varphi|\nabla u|(A \nabla \varphi) \cdot \nabla|\nabla u|-f^{\prime}(u)|\nabla u|^{2} \varphi^{2} d x \\
= & \int|\nabla u|^{2}(A \nabla \varphi) \cdot \nabla \varphi d x \\
& +\int_{\{\nabla u \neq 0\}} 2 \varphi|\nabla u|(A \nabla \varphi) \cdot \nabla|\nabla u|-\left(A \nabla u_{j}\right) \cdot \nabla\left(u_{j} \varphi^{2}\right) \\
& +\varphi^{2}(A \nabla|\nabla u|) \cdot \nabla|\nabla u| d x \\
= & \int|\nabla u|^{2}(A \nabla \varphi) \cdot \nabla \varphi d x+\int_{\{\nabla u \neq 0\}} a \varphi^{2}\left(|\nabla| \nabla u||^{2}-\sum_{j}\left|\nabla u_{j}\right|^{2}\right) \\
& -\left.a^{\prime} \varphi^{2}|\nabla u|\left|\nabla_{L}\right| \nabla u\right|^{2} d x .
\end{aligned}
$$

That is, using (1.5),

$$
\begin{aligned}
& \int|\nabla u|^{2}(A \nabla \varphi) \cdot \nabla \varphi d x \\
& \geq \int_{\{\nabla u \neq 0\}} \varphi^{2}\left[\left.\lambda_{1}\left|\nabla_{L}\right| \nabla u\right|^{2}\right. \\
& \left.\quad+\lambda_{2}\left(\sum_{j}\left|\nabla u_{j}\right|^{2}-\left.\left|\nabla_{L}\right| \nabla u\right|^{2}-|\nabla| \nabla u||^{2}\right)\right] d x .
\end{aligned}
$$

This and (2.9) imply the desired result.

Corollary 2.6. Let $N=2$ and $u \in C^{1}\left(\mathbb{R}^{2}\right) \cap C^{2}(\{\nabla u \neq 0\})$. Suppose that

$$
\begin{aligned}
& \int_{\{\nabla u \neq 0\}} {\left[\lambda_{1}(|\nabla u(x)|)\left|\nabla_{L_{u, x}}\right| \nabla u|(x)|^{2}\right.} \\
&\left.+\lambda_{2}(|\nabla u(x)|)|\nabla u(x)|^{2} \kappa_{1}^{2}(x)\right] \varphi^{2}(x) d x \\
& \leq K \int_{\mathbb{R}^{2}}|\nabla \varphi(x)|^{2} d x
\end{aligned}
$$

for some $K \geq 0$. 
Then,

$$
\kappa_{1}(x)=0
$$

and

$$
\nabla_{L_{u, x}}|\nabla u(x)|=0
$$

for any $x \in\{\nabla u \neq 0\}$. hold.

In particular, if $u$ fulfills the assumptions of Theorem 1.1, then (2.14) and (2.15)

Proof. First, we observe that (2.13) implies (2.14) and (2.15): this follows by fixing $R>0$, choosing

$$
\varphi:=\max \left\{0, \min \left\{1, \frac{\ln \left(R^{2} /|x|\right)}{\ln R}\right\}\right\}
$$

as test function in (2.13) and then sending $R \rightarrow+\infty$ (full details of this standard capacity argument are in [14]).

We now show that (2.14) and (2.15) hold under the assumptions of Theorem 1.1. Indeed, since $|\nabla u|$ is taken to be bounded in Theorem 1.1 and the map

$$
t \mapsto t^{2} \lambda_{1}(t)+t^{2} \lambda_{2}(t)
$$

belongs to $L_{\text {loc }}^{\infty}([0,+\infty))$, thanks to either (A1) or (A2), we have that

$$
|\nabla u|^{2}(A(\nabla u) \nabla \varphi) \cdot \nabla \varphi \leq K|\nabla \varphi|^{2}
$$

for any Lipschitz compactly supported $\varphi$, for a suitable $K>0$.

This and Theorem 2.5 yield that (2.13) is satisfied, thence (2.14) and (2.15) hold by what we have already proved.

\subsection{Level set analysis}

In what follows, we will consider connected components of the level sets (in the inherited topology).

Lemma 2.7. Let $v \in C^{1}\left(\mathbb{R}^{N}\right) \cap C^{2}(\{\nabla v \neq 0\})$. Fix $\bar{x} \in \mathbb{R}^{N}$.

Suppose that for any $x \in L_{v, \bar{x}} \cap\{\nabla v \neq 0\}$, we have that $\nabla_{L_{v, x}}|\nabla v(x)|=0$.

Then, $|\nabla v|$ is constant on every connected component of $L_{v, \bar{x}} \cap\{\nabla v \neq 0\}$.

Proof. Since any connected component of $L_{v, \bar{x}} \cap\{\nabla v \neq 0\}$ is a regular hypersurface, any two points in it may be joined by a $C^{1}$ path.

Let $L:=L_{v, \bar{x}}$ for short. We notice that, if $t_{1}>t_{0} \in \mathbb{R}$ and $\gamma \in C^{1}\left(\left[t_{0}, t_{1}\right], L \cap\right.$ $\{\nabla v \neq 0\})$, then

$$
\frac{d}{d t}|\nabla v(\gamma(t))|=\nabla|\nabla v(\gamma(t))| \cdot \dot{\gamma}(t)=\nabla_{L}|\nabla v(\gamma(t))| \cdot \dot{\gamma}(t)=0,
$$


thanks to (2.6). As a consequence,

$$
\begin{aligned}
& \text { if } \gamma \in C^{1}\left(\left[t_{0}, t_{1}\right], L \cap\{\nabla v \neq 0\}\right), \text { then } \\
& \qquad|\nabla v(\gamma(t))| \text { is constant for } t \in\left[t_{0}, t_{1}\right] .
\end{aligned}
$$

Now, we take $a$ and $b$ in $L \cap\{\nabla v \neq 0\}$ and $\gamma \in C^{1}([0,1], L)$ such that $\gamma(0)=a$ and $\gamma(1)=b$. Then $|\nabla v(a)|=|\nabla v(b)|$, by (2.16).

Corollary 2.8. Under the assumptions of Lemma 2.7, every connected component of $L_{v, x} \cap\{\nabla v \neq 0\}$ is closed in $\mathbb{R}^{N}$.

Proof. Let $M$ be any connected component of $L_{v, x} \cap\{\nabla v \neq 0\}$.

With no loss of generality, we suppose that $M \neq \emptyset$ and take $z \in M$.

Let $y \in \partial M$. Then there is a sequence $x_{n} \in M$ approaching $y$, thus

$$
v(y)=\lim _{n \rightarrow+\infty} v\left(x_{n}\right)=v(z) .
$$

Then, by Lemma 2.7, we have that $\left|\nabla v\left(x_{n}\right)\right|=|\nabla v(z)|$. So, since $z \in M$,

$$
|\nabla v(y)|=\lim _{n \rightarrow+\infty}\left|\nabla v\left(x_{n}\right)\right|=|\nabla v(z)| \neq 0 .
$$

By (2.17) and (2.18), we have that $y \in M$.

Corollary 2.9. Let the assumptions of Lemma 2.7 hold. Let $M$ be a connected component of $L_{v, x} \cap\{\nabla v \neq 0\}$. Suppose that $M \neq \emptyset$ and $M$ is contained in a hyperplane $\pi$. Then, $M=\pi$.

Proof. We show that

$$
M \text { is open in the topology of } \pi \text {. }
$$

For this, let $z \in M$. Then there exists an open set $O_{1}$ of $\mathbb{R}^{N}$ such that $z \in O_{1} \subset$ $\{\nabla v \neq 0\}$. Also, by the Implicit Function theorem, there exists an open set $O_{2}$ in $\mathbb{R}^{N}$ for which $z \in O_{2}$ and $L_{v, x} \cap O_{2}$ is a hypersurface. Since $M \subseteq \pi$, we have that $L_{v, x} \cap O_{2} \subseteq \pi$, hence $L_{v, x} \cap O_{2}$ is open in the topology of $\pi$.

Then, $z \in L_{v, x} \cap O_{1} \cap O_{2}$, which is an open set in $\pi$.

This proves (2.19).

Also, $M$ is closed in $\mathbb{R}^{N}$ and so $M=M \cap \pi$ is closed in $\pi$.

Hence, $M$ is open and closed in $\pi$.

Lemma 2.10. Let $v \in C^{1}\left(\mathbb{R}^{N}\right) \cap C^{2}(\{\nabla v \neq 0\})$ be such that $\nabla_{L_{v, x}}|\nabla v(x)|=0$ for every $x \in\{\nabla v \neq 0\}$.

Let $\bar{x} \in \mathbb{R}^{N}$.

Suppose that a non-empty connected component $\bar{L}$ of $L_{v, \bar{x}} \cap\{\nabla v \neq 0\}$ has zero principal curvatures at all points.

Then, $\bar{L}$ is a flat hyperplane. 
Proof. We use a standard differential geometry argument (see, for instance, [23, page 311]). Since the principal curvatures vanish identically, the normal of $\bar{L}$ is constant, thence $\bar{L}$ is contained in a hyperplane.

Then, the claim follows from Corollary 2.9.

Lemma 2.11. Let $v \in C^{1}\left(\mathbb{R}^{N}\right) \cap C^{2}(\{\nabla v \neq 0\})$. Suppose that

any connected component of $L_{v, x} \cap\{\nabla v \neq 0\}$

has zero principal curvatures at all points

and that

$$
\nabla_{L_{v, x}}|\nabla v(x)|=0
$$

for any $x \in\{\nabla v \neq 0\}$. Then, $v$ possesses one-dimensional symmetry, in the sense that there exists $\bar{v}: \mathbb{R} \rightarrow \mathbb{R}$ and $\omega \in S^{N-1}$ in such a way that $v(x)=\bar{v}(\omega \cdot x)$, for any $x \in \mathbb{R}^{N}$.

Proof. If $\nabla v(x)=0$ for any $x \in \mathbb{R}^{N}$, the one-dimensional symmetry is trivial.

If $\nabla v(\bar{x}) \neq 0$, then the connected component of $L_{v, \bar{x}} \cap\{\nabla v \neq 0\}$ passing through $\bar{x}$ is a hyperplane, due to Lemma 2.10 .

We observe that all these hyperplanes are parallel, because connected components cannot intersect.

Also, $v$ is constant on these hyperplanes, since each of them lies on a level set.

On the other hand, $v$ is also constant on any other possible hyperplane parallel to the ones of the above family, because the gradient vanishes identically there.

From this, the one-dimensional symmetry of $v$ follows by noticing that $v$ only depends on the orthogonal direction with respect to the above family of hyperplanes.

\subsection{Completion of the proof of Theorem 1.1}

We observe that $u$ satisfies (2.20) and (2.21), thanks to Corollary 2.6. Hence, the use of Lemma 2.11 ends the proof of Theorem 1.1.

\section{Optimality of the assumptions of Theorem 1.1}

We note that the assumption that $|\nabla u| \in L^{\infty}\left(\mathbb{R}^{N}\right)$ cannot be removed from Theorem 1.1, as the next observation points out:

Proposition 3.1. Let $\kappa>0$ and $\psi \in C^{1}((\kappa,+\infty))$ be such that

$$
\psi^{\prime}(t)>0
$$

for any $t>\kappa$, and

$$
\lim _{t \rightarrow+\infty} \psi(t)=+\infty
$$


Then, there exists $a \in C^{1}(0,+\infty)$ satisfying (1.2), (1.3) and (A2), and $u \in C^{2}\left(\mathbb{R}^{N}\right)$ which is a stable solution of

$$
\operatorname{div}(a(|\nabla u(x)|) \nabla u(x))-N=0
$$

and which satisfies

$$
|\nabla u(x)|=\psi(|x|)
$$

for any $|x|$ suitably large.

Also, $u$ does not possess one-dimensional symmetry.

Proof. Let $\kappa^{\prime \prime} \geq \kappa$ be such that $\kappa^{\prime}:=\psi\left(\kappa^{\prime \prime}\right)>0$ and let $\psi^{-1}$ be the inverse function of $\psi$.

Let $\phi \in C^{1}([0,+\infty))$ be such that $\phi(t):=\kappa^{\prime \prime} t / \kappa^{\prime}$ if $t \in\left[0, \kappa^{\prime} / 2\right], \phi(t):=$ $\psi^{-1}(t)$ if $t \in\left[\kappa^{\prime},+\infty\right)$, and $\phi^{\prime}(t)>0$ for any $t>0$.

Let $a(t):=\phi(t) / t$ for any $t>0$.

Then, $a$ clearly satisfies (1.2). Also,

$$
0<\phi^{\prime}(t)=a(t)+a^{\prime}(t) t
$$

thence (1.3) holds.

The fact that $a(t)=\kappa^{\prime \prime} / \kappa^{\prime}$ for $t \in\left(0, \kappa^{\prime} / 2\right)$ implies that (A2) is satisfied.

Let now

$$
v(r):=\int_{0}^{r} \phi^{-1}(s) d s
$$

and $u(x):=v(|x|)$.

Note that $v \in C^{2}(0,+\infty)$, and, since $\phi^{-1}(t)=\kappa^{\prime} t / \kappa^{\prime \prime}$ for small $t$, we have that $v^{\prime}\left(0^{+}\right)=0$ and $v^{\prime \prime}\left(0^{+}\right)=\kappa^{\prime} / \kappa^{\prime \prime}$.

Accordingly, $u \in C^{2}\left(\mathbb{R}^{N}\right)$, it satisfies (3.2) for $|x|>\kappa^{\prime}$ and it solves (3.1) by a straightforward calculation.

Since, in this case, $f^{\prime}$ vanishes, the stability condition (1.13) is also obviously satisfied.

Of course, $u$ does not possess one-dimensional symmetry, because it is radial and non-constant.

\section{Preliminaries for the proof of Theorem 1.2}

We take the assumptions of Theorem 1.2 and follow some ideas of [11]: namely, the proof of Theorem 1.2 will exploit a careful analysis at infinity, which reduces one dimension. Some ideas of $[1,2]$ will also be used for the energy estimates needed below.

Given a function $v: \mathbb{R}^{N} \rightarrow \mathbb{R}$, we set $v^{t}(x)=v^{t}\left(x^{\prime}, x_{N}\right):=v\left(x^{\prime}, x_{N}+t\right)$, for any $x=\left(x^{\prime}, x_{N}\right) \in \mathbb{R}^{N-1} \times \mathbb{R}$ and $t \in \mathbb{R}$. 


\subsection{Energy estimates}

Given $v: \mathbb{R}^{N} \rightarrow \mathbb{R}$, with $|\nabla v| \in L^{\infty}\left(\mathbb{R}^{N}\right)$, and $R>0$, we deal with the energy

$$
E_{R}(v):=\int_{B_{R}} \Lambda_{2}(|\nabla v(x)|)-F(v(x)) d x .
$$

The next result points out that the energy of a solution $v$ is controlled by the one of $v^{t}$, up to a term of order $R^{N-1}$ (in the course of the proof, we will see that such an additional term does not bother us, since $N=3$ in our case, and for large $t$ the energy of $v^{t}$ will be computed on one-dimensional profiles).

Lemma 4.1. Let $v \in C^{2}\left(\mathbb{R}^{N}\right)$ satisfy (1.1), with $\partial_{x_{N}} v \geq 0$ and $|v|+|\nabla v| \leq M$. There exists $C>0$, depending only on $N, M$ and $a$, in such a way that

$$
E_{R}(v) \leq E_{R}\left(v^{t}\right)+C R^{N-1}
$$

for any $t \in \mathbb{R}$.

Proof. The computations on [2, Section 2] may be easily adapted to our case, via the argument below. First,

$$
\begin{aligned}
\partial_{t} E_{R}\left(v^{t}\right) & =\int_{B_{R}} \lambda_{2}\left(\left|\nabla v^{t}\right|\right) \nabla v^{t} \cdot \partial_{t}\left(\nabla v^{t}\right)-f\left(v^{t}\right) \partial_{t} v^{t} d x \\
& =\int_{\partial B_{R}} a\left(\left|\nabla v^{t}\right|\right) \partial_{t} v^{t} \nabla v^{t} \cdot v d \sigma,
\end{aligned}
$$

where $v$ is the exterior normal of $B_{R}$, thanks to (1.1). Hence,

$$
\partial_{t} E_{R}\left(v^{t}\right) \geq-M^{\prime} \int_{\partial B_{R}} \partial_{t} v^{t}
$$

where

$$
M^{\prime}:=\sup _{0 \leq t \leq M}|t a(|t|)|,
$$

which is finite, thanks to (B1) or (B2). Therefore, if, say $T \geq 0$,

$$
\begin{aligned}
E_{R}\left(v^{T}\right)-E_{R}(v) & =\int_{0}^{T} \partial_{t} E_{R}\left(v^{t}\right) d t \\
& \geq-M^{\prime} \int_{0}^{T} \int_{\partial B_{R}} \partial_{t} v^{t} d \sigma d t=-M^{\prime} \int_{\partial B_{R}} \int_{0}^{T} \partial_{t} v^{t} d t d \sigma \\
& =-M^{\prime} \int_{\partial B_{R}}\left(v^{T}-v\right) d \sigma \geq-2 M M^{\prime}\left|\partial B_{R}\right|,
\end{aligned}
$$

as desired. 


\subsection{Elementary linear algebra}

Next is a straightforward consequence of (B1) or (B2) and Lemma 2.1:

Lemma 4.2. The maps $\xi \mapsto|A(\xi) \xi|$ and $\xi \mapsto|a(|\xi|) \xi|$ belong to $L_{\mathrm{loc}}^{\infty}\left(\mathbb{R}^{N} \backslash\{0\}\right)$ and they are continuously extended at the origin by attaining value zero.

If (B1) holds, there exists $c \in(0,1)$ such that

$$
c(\epsilon+|\xi|)^{p-2} \leq \lambda_{1}(\xi), \lambda_{2}(\xi) \leq \frac{1}{c}(\epsilon+|\xi|)^{p-2}
$$

for any $\xi \in \mathbb{R}^{N} \backslash\{0\}$.

If (B2) holds, given any $M>0$, there exists $c_{M} \in(0,1)$ such that

$$
c_{M} \leq \lambda_{1}(\xi), \lambda_{2}(\xi) \leq \frac{1}{c_{M}}
$$

for any $\xi \in \mathbb{R}^{N} \backslash\{0\}$ with $|\xi| \leq M$.

Corollary 4.3. We have that

$$
t^{2} a(t)-\Lambda_{2}(t)=\Lambda_{1}(t)
$$

for any $t>0$. Also, for any $M>0$, there exits $C_{M}>0$ such that

$$
t^{2} a(t) \leq C_{M} \Lambda_{2}(t)
$$

for any $t \in[0, M]$.

Proof. We observe that the function

$$
t \mapsto \Lambda_{1}(t)+\Lambda_{2}(t)-t^{2} a(t)
$$

is constant, due to (1.5) and (1.6), and

$$
\lim _{t \rightarrow 0^{+}} \Lambda_{i}(t)=\lim _{t \rightarrow 0^{+}} t^{2} a(t)=0,
$$

thanks to Lemma 4.2.

This proves (4.2).

By Lemma 4.2, we know that $\lambda_{1}$ and $\lambda_{2}$ are of the same order of magnitude, hence so are $\Lambda_{1}$ and $\Lambda_{2}$, by (1.6). This and (4.2) imply (4.3). 


\subsection{An elementary integral estimate}

For further reference, we point out the following simple observation:

Lemma 4.4. Let $\alpha \in \mathbb{R}$ and $\beta \in \mathbb{R}$. Suppose that $h \in C^{1}([\alpha, \beta])$ be such that $0<$ $\left|h^{\prime}\right| \leq M$. Then, there exists $C>0$, depending only on $M$ and $a$, in such a way that

$$
\int_{\alpha}^{\beta} \Lambda_{2}\left(h^{\prime}(t)\right) d t \leq C|h(\beta)-h(\alpha)| .
$$

Proof. By Lemma 4.2, if $0<|t| \leq M$,

$$
\frac{\Lambda_{2}(t)}{|t|}=\frac{1}{|t|} \int_{0}^{|t|} a(\tau) \tau d \tau \leq M^{\prime}
$$

for $M^{\prime}$ as in (4.1), and so

$$
\int_{\alpha}^{\beta} \Lambda_{2}\left(h^{\prime}(t)\right) d t \leq M^{\prime} \int_{\alpha}^{\beta}\left|h^{\prime}(t)\right| d t
$$

from which the desired claim easily follows, since $h$ is monotone.

\subsection{An elementary continuity property}

In our setting, $a$ and $A$ may not be well-defined at the origin and this may, in principle, cause problems when passing to the limit. The next observation is needed to avoid such complications:

Lemma 4.5. Suppose that $v_{j} \in C^{1}\left(\mathbb{R}^{N}\right)$ converges to some $v$ in $C_{\mathrm{loc}}^{1}\left(\mathbb{R}^{N}\right)$. Then,

$$
\begin{aligned}
\lim _{j \rightarrow+\infty} \int_{\mathbb{R}^{N}} a\left(\left|\nabla v_{j}\right|\right) \nabla v_{j} \cdot \nabla \phi & =\int_{\mathbb{R}^{N}} a(|\nabla v|) \nabla v \cdot \nabla \phi \\
\text { and } \lim _{j \rightarrow+\infty} \int_{\mathbb{R}^{N}} A\left(\nabla v_{j}\right) \nabla v_{j} \cdot \nabla \phi & =\int_{\mathbb{R}^{N}} A(\nabla v) \nabla v \cdot \nabla \phi,
\end{aligned}
$$

for any $\phi \in C_{0}^{\infty}\left(\mathbb{R}^{N}\right)$.

Proof. For short, we use here

$\alpha_{j}$ to denote either $a\left(\left|\nabla v_{j}(x)\right|\right) \nabla v_{j}(x)$ or $A\left(\nabla v_{j}(x)\right) \nabla v_{j}(x)$,

and $\alpha$ to denote either $a(|\nabla v(x)|) \nabla v(x)$ or $A(\nabla v(x)) \nabla v(x)$,

respectively.

By Lemma 4.2,

$$
\lim _{j \rightarrow+\infty} \alpha_{j}=0=\alpha
$$

if $x \in\{\nabla v=0\}$. 
Since $a$ and $A$ are continuous outside the origin, also

$$
\lim _{j \rightarrow+\infty} \alpha_{j}=\alpha
$$

if $x \in\{\nabla v \neq 0\}$.

Then, the Bounded Convergence theorem yields the desired result.

\subsection{ODE analysis}

We let $h \in C^{1}(\mathbb{R}) \cap W^{1, \infty}(\mathbb{R})$ be a weak solution of

$$
\left(a\left(\left|h^{\prime}(t)\right|\right) h^{\prime}(t)\right)^{\prime}+f(h(t))=0
$$

for any $t \in \mathbb{R}$.

This section is devoted to the analysis of the ODE in (4.4). For this purpose, it is enough to assume only that $f$ is continuous (such further generality may be useful in the applications, see, e.g. [15]).

First, we point out a regularity result. Namely, we estimate the Hölder exponent of $h^{\prime}$ (see Lemma 4.6), with the aim of proving that the map $t \rightarrow \Lambda_{1}\left(h^{\prime}(t)\right.$ ) is differentiable (see Corollary 4.7) and obtain a first integral of the motion (see Corollary 4.8).

Lemma 4.6. If (B1) holds with $\epsilon=0$, we have that $h \in C_{\mathrm{loc}}^{1,1 /(p-1)}(\mathbb{R}) \cap C^{2}\left(\left\{h^{\prime} \neq\right.\right.$ 0\}) if $p \geq 2$ and $h \in C^{2}(\mathbb{R})$ if $p<2$.

If either (B1) holds with $\epsilon>0$ or (B2) holds, we have that $h \in C_{\text {loc }}^{1,1}(\mathbb{R}) \cap$ $C^{2}\left(\left\{h^{\prime} \neq 0\right\}\right)$.

Proof. The most difficult case is when (B1) holds with $\epsilon=0$, which we consider first.

To this end, we set $\Phi(t):=t a(|t|)$ and $\gamma(t):=h^{\prime}(t) a\left(\left|h^{\prime}(t)\right|\right)$, for $t \in \mathbb{R}$.

Of course,

$$
\Phi\left(h^{\prime}(t)\right)=\gamma(t) .
$$

We also observe that $\gamma \in C(\mathbb{R})$ and, by (4.4), we have that $\gamma^{\prime}(t)=-f(h(t)) \in$ $C(\mathbb{R})$ in the distributional sense and, thence, as a function (see, e.g., [19, Theorem 6.10]). Accordingly,

$$
\gamma \in C^{1}(\mathbb{R})
$$

Moreover, by Lemma 4.2,

$$
c^{\star} t|t|^{p-2} \leq \Phi(t) \leq \frac{1}{c^{\star}} t|t|^{p-2}
$$

and, by Lemma 2.1,

$$
\Phi^{\prime}(t)=a(t)+t a^{\prime}(t)=\lambda_{1}(t)>0
$$

for any $t>0$. 
Since $\Phi$ is odd, (4.8) says that it is strictly increasing, hence invertible. Therefore, if $\Phi^{-1}$ is its inverse function, it is also odd, and we infer from (4.7) that

$$
c_{1} t|t|^{(2-p) /(p-1)} \leq \Phi^{-1}(t) \leq c_{2} t|t|^{(2-p) /(p-1)}
$$

for any $t>0$, where $c_{2}>c_{1}>0$ are appropriate constants.

Since $\Phi \in C^{1}(\mathbb{R} \backslash\{0\})$ by construction, thence $\Phi^{-1} \in C^{1}(\mathbb{R} \backslash\{0\})$, we deduce from the latter estimate that $\Phi^{-1} \in C^{1}(\mathbb{R})$ if $p<2$ and $\Phi^{-1} \in C^{0,1 /(p-1)}(\mathbb{R}) \cap$ $C^{1}(\mathbb{R} \backslash\{0\})$ if $p \geq 2$. Thence, by (4.5) and (4.6), we have that $h^{\prime}=\Phi^{-1} \circ \gamma$. This gives the desired regularity if (B1) holds with $\epsilon=0$.

In view of the fact that $h^{\prime} \in L^{\infty}(\mathbb{R})$ and recalling footnote 4 , we see that this also gives the desired claim when (B2) holds.

Case (B1) with $\epsilon>0$ reduces to the case $p=2$.

This concludes the proof of Lemma 4.6.

Corollary 4.7. Let $L_{1}(t):=\Lambda_{1}\left(h^{\prime}(t)\right)$. Then, we have that $L_{1} \in C(\mathbb{R}) \cap C^{1}\left(\left\{h^{\prime} \neq\right.\right.$ $0\})$, that $L_{1}$ is differentiable on $\mathbb{R}$ and that $L_{1}^{\prime}\left(t^{\star}\right)=0$ if $h^{\prime}\left(t^{\star}\right)=0$.

Proof. We first consider the case in which (B1) holds with $\epsilon=0$. Then, exploiting Lemmata 2.1 and 4.2, (1.5) and (1.6), we see that

$$
\begin{aligned}
\left|\Lambda_{1}(t)\right| & \leq \int_{0}^{|t|}\left|a(\tau)+a^{\prime}(\tau) \tau\right| \tau d \tau \\
& \leq \text { const } \int_{0}^{|t|} \tau^{p-1} d \tau \\
& \leq \text { const }|t|^{p},
\end{aligned}
$$

for $|t| \leq 1$.

Consequently, for small $s$, if $t^{\star} \in\left\{h^{\prime}=0\right\}$,

$$
\begin{aligned}
\left|\frac{L_{1}\left(t^{\star}+s\right)-L_{1}\left(t^{\star}\right)}{s}\right| & =\left|\frac{\Lambda_{1}\left(h^{\prime}\left(t^{\star}+s\right)\right)}{s}\right| \\
& \leq \frac{\text { const }\left|h^{\prime}\left(t^{\star}+s\right)\right|^{p}}{|s|} \\
& =\frac{\operatorname{const}\left|h^{\prime}\left(t^{\star}+s\right)-h^{\prime}\left(t^{\star}\right)\right|^{p}}{|s|} .
\end{aligned}
$$

So, by Lemma 4.6,

$$
\left|\frac{L_{1}\left(t^{\star}+s\right)-L_{1}\left(t^{\star}\right)}{s}\right| \leq \frac{\text { const }|s|^{p /(p-1)}}{|s|}=\mathrm{const}|s|^{1 /(p-1)},
$$

if $p \geq 2$, while

$$
\left|\frac{L_{1}\left(t^{\star}+s\right)-L_{1}\left(t^{\star}\right)}{s}\right| \leq \frac{\text { const }|s|^{p}}{|s|}=\mathrm{const}|s|^{p-1},
$$

if $1<p<2$.

By sending $s \rightarrow 0$, we see that $L_{1}^{\prime}\left(t^{\star}\right)=0$. 
We now deal with the case in which either (B1) holds with $\epsilon>0$ or (B2) holds. In these circumstances, the eigenvalues of $A$ are locally bounded, due to Lemma 4.2, and therefore, by Lemma 2.1, (1.5) and (1.6), we conclude that

$$
\begin{aligned}
\left|\Lambda_{1}(t)\right| & \leq \int_{0}^{|t|}\left|a(\tau)+a^{\prime}(\tau) \tau\right| \tau d \tau \\
& \leq \text { const } \int_{0}^{|t|} \tau d \tau \\
& \leq \text { const }|t|^{2},
\end{aligned}
$$

for $|t| \leq 1$ and so, for small $s$,

$$
\begin{aligned}
& \left|\frac{L_{1}\left(t^{\star}+s\right)-L_{1}\left(t^{\star}\right)}{s}\right|=\left|\frac{\Lambda_{1}\left(h^{\prime}\left(t^{\star}+s\right)\right)}{s}\right| \\
& \leq \frac{\text { const }\left|h^{\prime}\left(t^{\star}+s\right)\right|^{2}}{|s|}=\frac{\text { const }\left|h^{\prime}\left(t^{\star}+s\right)-h^{\prime}\left(t^{\star}\right)\right|^{2}}{|s|} \\
& \leq \text { const }|s|,
\end{aligned}
$$

due to Lemma 4.6. By sending $s \rightarrow 0$, we see that $L_{1}^{\prime}\left(t^{\star}\right)=0$ in this case too.

Corollary 4.8. For any $t, s \in \mathbb{R}$,

$$
\Lambda_{1}\left(h^{\prime}(t)\right)+F(h(t))=\Lambda_{1}\left(h^{\prime}(s)\right)+F(h(s)) .
$$

Proof. If $h \in C^{2}(t-b, t+b)$, with $h^{\prime} \neq 0$ in $(t-b, t+b)$, for some $b>0$, we have that

$$
\begin{aligned}
& \frac{d}{d t}\left(\Lambda_{1}\left(h^{\prime}(t)\right)+F(h(t))\right) \\
& =\left(a\left(\left|h^{\prime}(t)\right|\right) h^{\prime}(t)+a^{\prime}\left(\left|h^{\prime}(t)\right|\right)\left|h^{\prime}(t)\right| h^{\prime}(t)\right) h^{\prime \prime}(t)+f(h(t)) h^{\prime}(t) \\
& =\left[\left(a\left(\left|h^{\prime}(t)\right|\right)+a^{\prime}\left(\left|h^{\prime}(t)\right|\right)\left|h^{\prime}(t)\right|\right) h^{\prime \prime}(t)+f(h(t))\right] h^{\prime}(t) \\
& =\left[\left(a\left(\left|h^{\prime}(t)\right|\right) h^{\prime}(t)\right)^{\prime}+f(h(t))\right] h^{\prime}(t)=0,
\end{aligned}
$$

due to (4.4).

On the other hand, if $h^{\prime}\left(t^{\star}\right)=0$, we deduce from Corollary 4.7 that

$$
\left.\frac{d}{d t}\left(\Lambda_{1}\left(h^{\prime}(t)\right)+F(h(t))\right)\right|_{t=t^{\star}}=L_{1}^{\prime}\left(t^{\star}\right)+f\left(h\left(t^{\star}\right)\right) h^{\prime}\left(t^{\star}\right)=0+0,
$$

as desired. 
Corollary 4.9. For any $t \in \mathbb{R}$,

$$
\Lambda_{1}\left(h^{\prime}(t)\right)+F(h(t))=F(\inf h)=F(\sup h) .
$$

Proof. We only deal with $\inf h$, since sup $h$ may be dealt with analogously.

Let us first consider the case in which inf $h$ is attained at some point $\bar{t} \in \mathbb{R}$. Then, from Corollary 4.8,

$$
\Lambda_{1}\left(h^{\prime}(t)\right)+F(h(t))=\Lambda_{1}(0)+F(h(\bar{t}))=0+F(\inf h),
$$

as desired.

Suppose now that inf $h$ is not attained and take a sequence $t_{n} \rightarrow+\infty$ such that $h\left(t_{n}\right) \rightarrow \inf h$. Let us consider $h_{n}(t):=h\left(t+t_{n}\right)$. Since $\left\|h^{\prime}\right\|_{C^{1, \alpha}(\mathbb{R})}$ is finite (see, e.g., formulas (4.1) and (4.2) in [7]), we have that $h_{n}$, up to subsequence, converges to a suitable $\widetilde{h}$ in $C_{\text {loc }}^{1}(\mathbb{R})$ and therefore $\widetilde{h}$ is also a weak solution of (4.4), due to Lemma 4.5. Since

$$
\tilde{h}(0)=\lim _{n \rightarrow+\infty} h\left(t_{n}\right)=\inf h \leq \lim _{n \rightarrow+\infty} h\left(t+t_{n}\right)=\widetilde{h}(t)
$$

we have that $\inf \widetilde{h}$ is attained and, in fact, it agrees with $\widetilde{h}(0)=\inf h$. So, for what we have already proved and Corollary 4.8 ,

$$
\begin{aligned}
F(\inf h) & =F(\inf \tilde{h}) \\
& =\Lambda_{1}\left(\tilde{h}^{\prime}(t)\right)+F(\tilde{h}(t)) \\
& =\lim _{n \rightarrow+\infty} \Lambda_{1}\left(h^{\prime}\left(t+t_{n}\right)\right)+F\left(h\left(t+t_{n}\right)\right) \\
& =\lim _{n \rightarrow+\infty} \Lambda_{1}\left(h^{\prime}(t)\right)+F(h(t)) \\
& =\Lambda_{1}\left(h^{\prime}(t)\right)+F(h(t)),
\end{aligned}
$$

as desired.

We remark that, as a consequence of Corollary 4.9,

$$
F(\inf h)=F(\sup h)=F\left(h\left(t^{\star}\right)\right)
$$

for any $t^{\star} \in\left\{h^{\prime}=0\right\}$.

We now classify the solution of the ODE in (4.4):

Lemma 4.10. One of the following possibilities holds:

I. $h$ is constant,

II. $\left\{h^{\prime}=0\right\}=\emptyset$,

III. $h^{\prime}(t) \neq 0$ for any $t$ in a bounded interval of the form $\left(\beta_{1}, \beta_{2}\right)$ with $h^{\prime}\left(\beta_{1}\right)=$ $h^{\prime}\left(\beta_{2}\right)=0$ and

$$
F\left(h\left(\beta_{1}\right)\right)=F\left(h\left(\beta_{2}\right)\right)=F(\inf h)=F(\sup h) .
$$


IV. $h^{\prime}(t) \neq 0$ for any $t$ in an unbounded interval either of the form $(\beta,+\infty)$ or $(-\infty, \beta)$, with $\beta \in \mathbb{R}, h^{\prime}(\beta)=0$ and

$$
F(h(\beta))=F(\inf h)=F(\sup h) .
$$

Proof. Suppose that I does not hold. Then there exists some $t^{\star}$ in such a way that $h^{\prime}\left(t^{\star}\right) \neq 0$. Take an interval $I$ around $t^{\star}$, as large as possible, in such a way that $h^{\prime}(t) \neq 0$ for $t \in I$. If $I=\mathbb{R}$, we are in case II. If $I$ is bounded, say with extrema $\beta_{1}<\beta_{2}$, we have that $h^{\prime}\left(\beta_{1}\right)=h^{\prime}\left(\beta_{2}\right)=0$ and so we are in case III and (4.10) follows from (4.9).

The latter case is that $I$ is unbounded in one direction, meaning that $I$ is either $(\beta,+\infty)$ or $(-\infty, \beta)$. Then, $h^{\prime}(\beta)=0$ and we are in case IV: thus, (4.11) follows again from (4.9).

\subsection{A pointwise energy estimate}

We now recall a pointwise energy estimate for weak solutions of (1.1). The first estimate of this type was given in [20] for the standard phase transition model and extensions to more general cases are in [5] and [7]. In our setting, such a result translates into:

Lemma 4.11. Suppose that $u \in C^{1}\left(\mathbb{R}^{N}\right) \cap W^{1, \infty}\left(\mathbb{R}^{N}\right)$ is a weak solution of (1.1). Let

$$
c_{u}:=\sup _{t \in[\inf u, \sup u]} F(t) .
$$

Then,

$$
\Lambda_{1}(|\nabla u(x)|) \leq c_{u}-F(u(x))
$$

for any $x \in \mathbb{R}^{N}$.

Proof. We will make use ${ }^{6}$ of [5]. The quantities called $f, \Phi(s)$ and $F$ in [5] correspond here to $-f, 2 \Lambda_{2}(\sqrt{s})$ and $c_{u}-F$, respectively. Accordingly, the quantity $a_{i j}$ of [5] agrees with the one denoted here by $A_{i j}$ and the function

$$
P(u ; x)=2 \Phi^{\prime}\left(|\nabla u(x)|^{2}\right)|\nabla u(x)|^{2}-\Phi\left(|\nabla u(x)|^{2}\right)-2 F(u(x))
$$

introduced in (2.1) of [5] becomes here

$$
\begin{aligned}
& 2 a(|\nabla u(x)|)|\nabla u(x)|^{2}-2 \Lambda_{2}(|\nabla u(x)|)+2\left(F(u(x))-c_{u}\right) \\
& =2\left(\Lambda_{1}(|\nabla u(x)|)+F(u(x))-c_{u}\right) .
\end{aligned}
$$

thanks to (4.2).

6 It would also be possible to use here [7, Corollary 4.9]. To compare notations, what in [7] is called $\Phi(\xi, \sigma)$ reduces here to $\Lambda_{2}(|\sigma|)-F(\xi)$. Accordingly, the quantity $G(\xi, s)$ given in (1.5) of [7] is here $2 \Lambda_{2}(\sqrt{s})-2 F(\xi)$ for any $s>0$, and so $G_{s}(\xi, s)$ agrees here with $a(\sqrt{s})$. Also, if $G^{u}$ is the quantity introduced in Corollary 4.9 of [7], we have that $G^{u}=-2 c_{u}$ here. Then, our claim would follow from (4.41) in [7] and (4.2).

We have preferred, however, to follow [5] in detail, in order to impose a slightly weaker regularity assumption on $a$, namely (1.16). 
Then, [5, Theorem 1.6] states that $P(u ; x) \leq 0$, thence the desired claim.

But a caveat has to be taken into account in order to use [5, Theorem 1.6]. Namely, the functional in [5] was assumed to be smooth, namely $F \in C^{2}(\mathbb{R})$ and $\Phi \in C^{3}((0,+\infty))$, in order to perform the computations of Theorem 2.2 there in the classical sense, while $F$ and $\Phi$ are here only in $C_{\text {loc }}^{1,1}(\mathbb{R})$ and $C_{\text {loc }}^{2,1}((0,+\infty))$, by (1.16). This, however, is enough to repeat the computations of [5, Theorem 2.2] verbatim in the weak distributional sense, and hence to get the desired result.

\subsection{Analysis at infinity}

The solutions which are monotone in one direction, say in the $N$ th direction for definiteness, may exhibit a particularly nice behavior when $x_{N} \rightarrow \pm \infty$.

The reason for this is that the functions, for $x_{N} \rightarrow \pm \infty$, depend on one variable less and so, in some sense, the symmetry results (or, in our technique, the level set estimates) obtained in $\mathbb{R}^{N-1}$ may be of some use.

This idea goes back to [2] and variations of it have been used by several authors.

The main result we need is the following, in which the solutions at infinity are shown to satisfy the Poincare type formula of Theorem 2.5, in one dimension less:

Theorem 4.12. Let $u \in C^{1}\left(\mathbb{R}^{N}\right) \cap W^{1, \infty}\left(\mathbb{R}^{N}\right)$ be a stable weak solution of $(1.1)$ in $\mathbb{R}^{N}$.

Suppose that

$$
\{\nabla u=0\}=\emptyset
$$

and

$$
\partial_{x_{N}} u \geq 0 .
$$

Then, the limits

$$
\begin{aligned}
& \underline{u}\left(x^{\prime}\right):=\lim _{t \rightarrow-\infty} u\left(x^{\prime}, x_{N}+t\right) \\
& \bar{u}\left(x^{\prime}\right):=\lim _{t \rightarrow+\infty} u\left(x^{\prime}, x_{N}+t\right)
\end{aligned}
$$

exist and are finite for any $x^{\prime} \in \mathbb{R}^{N-1}$.

Also,

$$
\text { the limits in (4.14) hold in } C_{\mathrm{loc}}^{1}\left(\mathbb{R}^{N}\right)
$$

and

$$
\underline{u} \text { and } \bar{u} \text { are weak solutions of (1.1) in } \mathbb{R}^{N-1} \text {. }
$$


Finally,

$$
\begin{gathered}
\int_{\mathbb{R}^{N-1} \cap\{\nabla \bar{u} \neq 0\}}\left[\left.\lambda_{1}(|\nabla \bar{u}|)\left|\nabla_{L_{\bar{u}, x^{\prime}}}\right| \nabla \bar{u}\right|^{2}\right. \\
\left.+\lambda_{2}(|\nabla \bar{u}|)|\nabla \bar{u}|^{2} \sum_{\ell=1}^{N-2} \kappa_{\ell, \bar{u}}^{2}\right] \phi^{2} d x^{\prime} \\
\leq \int_{\mathbb{R}^{N-1}}|\nabla \bar{u}|^{2}(\tilde{A}(\nabla \bar{u}) \nabla \phi) \cdot \nabla \phi d x^{\prime}
\end{gathered}
$$

for any locally Lipschitz, compactly supported $\phi: \mathbb{R}^{N-1} \rightarrow \mathbb{R}$ (and an analogous claim holds for $\underline{u}$ ).

Here above, we have used $\tilde{A} \in \operatorname{Mat}((N-1) \times(N-1))$ defined by

$$
\tilde{A}_{i j}=A_{i j} \quad \text { for } 1 \leq i, j \leq N-1
$$

Proof. We recall that

$$
\|u\|_{C^{1, \beta}\left(B_{R}\right)} \leq C_{R}
$$

for some $\beta>0$. Also, given $\delta>0$, if $\Omega \subset\{|\nabla u| \geq \delta\}$ and $\operatorname{diam}(\Omega) \leq R$,

$$
\|u\|_{C^{2, \beta_{\delta, R}(\Omega)}} \leq C_{\delta, R},
$$

for some $\beta_{\delta, R} \in(0,1)$ by Schauder Estimates: for details, we refer to [7, page 459] and to ${ }^{7}$ the bibliography quoted there.

Since $u$ is bounded and monotone in the $N$ th direction, due to (4.13), the limits in (4.14) exist and are finite.

Then, (4.15) follows from (4.18) and the theorem of Ascoli.

We now fix $\delta>0$. Let $B$ a ball contained in

$$
\mathcal{D}_{\delta}:=\left\{\left(x^{\prime}, x_{N}\right) \in \mathbb{R}^{N-1} \times \mathbb{R} \text { such that }\left|\nabla \bar{u}\left(x^{\prime}\right)\right| \geq \delta\right\} .
$$

7 The constants $\beta$ and $C_{R}$ in (4.18) depend on $\|u\|_{W^{1, \infty}\left(\mathbb{R}^{N}\right)}$. More precisely, if (B1) holds, then we use (4.1) and (4.2) in [7] to obtain

$$
\begin{aligned}
& \|\nabla u\|_{L^{\infty}\left(\mathbb{R}^{N}\right)} \leq M \text { and } \\
& \sup _{x, y \in B_{R}}|\nabla u(x)-\nabla u(y)| \leq \frac{M}{R^{\gamma}}\left(\frac{|x-y|}{R}\right)^{\beta}
\end{aligned}
$$

for suitable $M, \gamma$ and $\beta$ possibly depending on $\|u\|_{L^{\infty}\left(\mathbb{R}^{N}\right)}$.

If, on the other hand, (B2) holds, the equation is uniformly elliptic, because $\|\nabla u\|_{L^{\infty}\left(\mathbb{R}^{N}\right)}<+\infty$ and then the regularity of $u$ follows from Schauder Estimates.

In any case, this bounds the constants $\beta$ and $C_{R}$ in (4.18) in dependence of $\|u\|_{W^{1, \infty}\left(\mathbb{R}^{N}\right)}$.

Thus, the constants $\beta_{\delta, R}$ and $C_{\delta, R}$ in (4.19) also depend on $\|u\|_{W^{1, \infty}\left(\mathbb{R}^{N}\right)}$. The dependence of such constants upon $\delta$ is due to the uniform ellipticity of the equation in $\{|\nabla u| \geq \delta\}$. 
It follows from (4.15) that $\left|\nabla u\left(x^{\prime}, x_{N}+t\right)\right| \geq \delta / 2$ if $\left(x^{\prime}, x_{N}\right) \in B$ and $t \geq t_{\delta}$, as long as $t_{\delta}$ is suitably large.

So, by (4.19), the second limit in (4.14) holds in $C^{2}(B)$.

That is,

$$
\text { the second limit in (4.14) holds in } C_{\text {loc }}^{2}\left(\mathcal{D}_{\delta}\right) \text {, }
$$

and an analogous result holds for the first limit.

We also observe that (4.15) and (4.20) imply that $\bar{u} \in C_{\mathrm{loc}}^{1, \beta}\left(\mathbb{R}^{N-1}\right) \cap C^{2}(\{\nabla \bar{u} \neq$ $0\}$ ) for some $\beta \in(0,1)$, and that

$$
\nabla_{L_{\bar{u}, x^{\prime}}}|\nabla \bar{u}|\left(x^{\prime}\right)=\lim _{t \rightarrow+\infty} \nabla_{L_{u,\left(x^{\prime}, x_{N}+t\right)}}|\nabla u|\left(x^{\prime}, x_{N}+t\right)
$$

for any $x^{\prime} \in\{|\nabla \bar{u}| \geq \delta\}$, due to (2.6).

What is more, if $\bar{\kappa}_{\ell}\left(y^{\prime}\right)=\kappa_{\ell, \bar{u}}\left(y^{\prime}\right)$ are the principal curvatures of $L_{\bar{u}, x^{\prime}}$ at $y^{\prime} \in$ $L_{\bar{u}, x^{\prime}} \cap\{|\nabla \bar{u}| \geq \delta\}$, for $\ell=1, \ldots, N-2$, a consequence of (2.9), (4.15) and (4.20) is that

$$
\left|\nabla \bar{u}\left(x^{\prime}\right)\right|^{2} \sum_{\ell=1}^{N-2} \bar{\kappa}_{\ell}^{2}\left(y^{\prime}\right)=\lim _{t \rightarrow+\infty}\left|\nabla u\left(x^{\prime}, x_{N}+t\right)\right|^{2} \sum_{\ell=1}^{N-1} \kappa_{\ell}^{2}\left(y^{\prime}, x_{N}+t\right) .
$$

Of course, statements analogous to (4.21) and (4.22) also hold for $\underline{u}$.

Moreover,

$$
\int_{\mathbb{R}^{N}} a(|\nabla u|) \nabla u \cdot \nabla \varphi-f(u) \varphi d x=0,
$$

for any $\varphi \in C_{0}^{\infty}\left(\mathbb{R}^{N}\right)$, due to (1.1).

We take $\varphi:=\varphi_{1}\left(x^{\prime}\right) \varphi_{2}\left(x_{N}\right)$, with $\varphi \in C_{0}^{\infty}\left(\mathbb{R}^{N-1}\right)$ and $\varphi_{2} \in C_{0}^{\infty}(\mathbb{R})$, with

$$
\int_{\mathbb{R}} \varphi_{2}\left(x_{N}\right) d x_{N}=1
$$

By passing (4.23) to the limit and recalling (4.15) and Lemma 4.5, we deduce that

$$
\int_{\mathbb{R}^{N-1}} a\left(\left|\nabla \bar{u}\left(x^{\prime}\right)\right|\right) \nabla \bar{u}\left(x^{\prime}\right) \cdot \nabla \varphi_{1}\left(x^{\prime}\right)-f\left(\bar{u}\left(x^{\prime}\right)\right) \varphi_{1}\left(x^{\prime}\right) d x^{\prime}=0,
$$

for any $\varphi_{1} \in C_{0}^{\infty}\left(\mathbb{R}^{N-1}\right)$, and the same for $\underline{u}$, thus proving (4.16).

Furthermore, $u$ satisfies (2.10), by Theorem 2.5, hence so does $u^{t}$. We then take $\varphi:=\varphi_{1}\left(x^{\prime}\right) \varphi_{2}\left(x_{N}\right)$, with $\varphi_{1}: \mathbb{R}^{N-1} \rightarrow \mathbb{R}$ and $\varphi_{2}: \mathbb{R} \rightarrow \mathbb{R}$ locally Lipschitz, compactly supported functions. We also take $\varphi_{2}$ of the form $\varphi_{2}\left(x_{N}\right):=$ $\sqrt{\mu} \tau\left(\mu x_{N}\right)$, where $\mu>0$ is a small parameter, $\tau \in C_{0}^{\infty}(\mathbb{R})$ and

$$
\int_{\mathbb{R}} \tau^{2}\left(x_{N}\right) d x_{N}=1
$$


We thus have that

$$
\int_{\mathbb{R}} \varphi_{2}^{2}\left(x_{N}\right) d x_{N}=1
$$

Now, we pass (2.10) to the limit. Namely, we set $u^{t}(x):=u\left(x^{\prime}, x_{N}+t\right)$ and $\kappa_{\ell}^{t}(x):=\kappa_{\ell, u^{t}}(x)=\kappa_{\ell, u}\left(x^{\prime}, x_{N}+t\right)$, and we use (2.10), (4.12), (4.15), (4.21), (4.22), (4.24) and Lemma 4.5 to obtain

$$
\begin{aligned}
& \int_{\{|\nabla \bar{u}| \geq \delta\}}\left[\lambda_{1}(|\nabla \bar{u}|)\left|\nabla_{L_{\bar{u}, x^{\prime}}}\right| \nabla \bar{u}||^{2}+\lambda_{2}(|\nabla \bar{u}|)|\nabla \bar{u}|^{2} \sum_{\ell=1}^{N-2} \bar{\kappa}_{\ell}^{2}\right] \varphi_{1}^{2} d x^{\prime} \\
& =\int_{\mathbb{R}} \int_{\{|\nabla \bar{u}| \geq \delta\}}\left[\left.\lambda_{1}(|\nabla \bar{u}|)\left|\nabla_{L_{\bar{u}, x^{\prime}}}\right| \nabla \bar{u}\right|^{2}+\lambda_{2}(|\nabla \bar{u}|)|\nabla \bar{u}|^{2} \sum_{\ell=1}^{N-2} \bar{\kappa}_{\ell}^{2}\right] \varphi^{2} d x^{\prime} d x_{N} \\
& =\lim _{t \rightarrow+\infty} \int_{\mathbb{R}} \int_{\{|\nabla \bar{u}| \geq \delta\}}\left[\left.\lambda_{1}\left(\left|\nabla u^{t}\right|\right)\left|\nabla_{L_{u^{t}, x}}\right| \nabla u^{t}\right|^{2}\right. \\
& \left.+\lambda_{2}\left(\left|\nabla u^{t}\right|\right)\left|\nabla u^{t}\right|^{2} \sum_{\ell=1}^{N-1}\left(\kappa_{\ell}^{t}\right)^{2}\right] \varphi^{2} d x^{\prime} d x_{N} \\
& =\lim _{t \rightarrow+\infty} \int_{\mathcal{D}_{\delta}}\left[\left.\lambda_{1}\left(\left|\nabla u^{t}\right|\right)\left|\nabla_{L_{u^{t}, x}}\right| \nabla u^{t}\right|^{2}\right. \\
& \left.+\lambda_{2}\left(\left|\nabla u^{t}\right|\right)\left|\nabla u^{t}\right|^{2} \sum_{\ell=1}^{N-1}\left(\kappa_{\ell}^{t}\right)^{2}\right] \varphi^{2} d x \\
& \leq \lim _{t \rightarrow+\infty} \int_{\mathbb{R}^{N}}\left|\nabla u^{t}\right|^{2}\left(A\left(\nabla u^{t}\right) \nabla \varphi\right) \cdot \nabla \varphi d x=\int_{\mathbb{R}^{N}}|\nabla \bar{u}|^{2}(A(\nabla \bar{u}) \nabla \varphi) \cdot \nabla \varphi d x .
\end{aligned}
$$

Thence, by taking $\delta$ arbitrarily small,

$$
\begin{aligned}
& \int_{\{\nabla \bar{u} \neq 0\}}\left[\lambda_{1}(|\nabla \bar{u}|)\left|\nabla_{L_{\bar{u}, x^{\prime}}}\right| \nabla \bar{u}||^{2}+\lambda_{2}(|\nabla \bar{u}|)|\nabla \bar{u}|^{2} \sum_{\ell=1}^{N-2} \bar{\kappa}_{\ell}^{2}\right] \varphi_{1}^{2} d x^{\prime} \\
& \leq \int_{\mathbb{R}^{N}}|\nabla \bar{u}|^{2}(A(\nabla \bar{u}) \nabla \varphi) \cdot \nabla \varphi d x .
\end{aligned}
$$

We now observe that $\partial_{x_{N}} \bar{u}=0$ and so

$$
|\nabla \bar{u}|^{2} A_{i j}(\nabla \bar{u})=|\nabla \bar{u}|^{2} a(|\bar{u}|) \delta_{i j} \quad \text { if either } i=N \text { or } j=N .
$$

Therefore,

$$
\begin{aligned}
& |\nabla \bar{u}|^{2}(A(\nabla \bar{u}) \nabla \varphi) \cdot \nabla \varphi \\
& =|\nabla \bar{u}|^{2}\left(\tilde{A}(\nabla \bar{u})\left(\partial_{x_{1}} \varphi, \ldots, \partial_{x_{N-1}} \varphi\right)\right) \cdot\left(\partial_{x_{1}} \varphi, \ldots, \partial_{x_{N-1}} \varphi\right) \\
& \quad+|\nabla \bar{u}|^{2} a(|\nabla \bar{u}|)\left|\partial_{x_{N}} \varphi\right|^{2} .
\end{aligned}
$$


This, Lemma 4.2, (4.24) and (4.25) imply that

$$
\begin{aligned}
& \int_{\{\nabla \bar{u} \neq 0\}}\left[\lambda_{1}(|\nabla \bar{u}|)\left|\nabla_{L_{\bar{u}, x^{\prime}}}\right| \nabla \bar{u}||^{2}+\lambda_{2}(|\nabla \bar{u}|)|\nabla \bar{u}|^{2} \sum_{\ell=1}^{N-2} \bar{\kappa}_{\ell}^{2}\right] \varphi_{1}^{2} d x^{\prime} \\
& \leq \int_{\mathbb{R}^{N}} \varphi_{2}^{2}\left(x_{N}\right)|\nabla \bar{u}|^{2}\left(\tilde{A}(\nabla \bar{u}) \nabla \varphi_{1}\right) \cdot \nabla \varphi_{1} d x \\
& \quad+\text { const } \int_{\mathbb{R}^{N}} \varphi_{1}^{2}\left(x^{\prime}\right)\left|\varphi_{2}^{\prime}\left(x_{N}\right)\right|^{2} d x \\
& \leq \quad \int_{\mathbb{R}^{N}} \tau^{2}\left(\mu x_{N}\right)\left|\nabla \bar{u}\left(x^{\prime}\right)\right|^{2}\left(\tilde{A}\left(\nabla \bar{u}\left(x^{\prime}\right)\right) \nabla \varphi_{1}\left(x^{\prime}\right)\right) \cdot \nabla \varphi_{1}\left(x^{\prime}\right) d x^{\prime} d x_{N} \\
& \quad+\text { const } \mu^{3} \int_{\mathbb{R}^{N}} \varphi_{1}^{2}\left(x^{\prime}\right)\left|\tau^{\prime}\left(\mu x_{N}\right)\right|^{2} d x^{\prime} d x_{N} \\
& \leq \int_{\mathbb{R}^{N-1}}\left|\nabla \bar{u}\left(x^{\prime}\right)\right|^{2}\left(\tilde{A}\left(\nabla \bar{u}\left(x^{\prime}\right)\right) \nabla \varphi_{1}\left(x^{\prime}\right)\right) \cdot \nabla \varphi_{1}\left(x^{\prime}\right) d x^{\prime} \\
& \quad+\operatorname{const} \mu^{2} \int_{\mathbb{R}^{\prime}}\left|\tau^{\prime}(s)\right|^{2} d s \cdot \int_{\mathbb{R}^{N-1}} \varphi_{1}^{2}\left(x^{\prime}\right) d x^{\prime},
\end{aligned}
$$

where the above constants possibly depend on $\|\bar{u}\|_{C^{1}\left(\mathbb{R}^{N}\right)}$. We then conclude that (4.17) holds by sending $\mu \rightarrow 0^{+}$.

In the notation of (4.14), it is convenient to define

$$
\begin{array}{rlrl}
M & :=\sup _{\mathbb{R}^{N}} u, & \underline{M}:=\sup _{\mathbb{R}^{N-1}} \underline{u}, & \bar{M}:=\sup _{\mathbb{R}^{N-1}} \bar{u}, \\
m:=\inf _{\mathbb{R}^{N}} u, & \underline{m}:=\inf _{\mathbb{R}^{N-1}} \underline{u}, & \bar{m}:=\inf _{\mathbb{R}^{N-1}} \bar{u}
\end{array}
$$

and to observe that, from (4.13),

$$
\bar{M}=M \geq \underline{M} \text { and } \bar{m} \geq m=\underline{m} .
$$

Recalling the notation in Lemma 4.11, we write

$$
c_{u}:=\sup _{[m, M]} F
$$

In our case, this quantity may be better determined:

Lemma 4.13. Let $u \in C^{1}\left(\mathbb{R}^{N}\right) \cap W^{1, \infty}\left(\mathbb{R}^{N}\right)$ be a weak solution of $(1.1)$ with $\{\nabla u=$ $0\}=\emptyset$. Then, we have that $c_{u}>F(t)$ for any $t \in(m, M)$. In particular, $c_{u}=$ $\max \{F(m), F(M)\}$ and if $F\left(t^{\star}\right)=c_{u}$ then $t^{\star} \in\{m, M\}$. 
Proof. The second and third claims are consequence of the first one, which we now prove. Suppose, by contradiction, that $c_{u}=F(t)$ for some $t \in(m, M)$. Then, there exists $x^{\star} \in \mathbb{R}^{N}$ in such a way that $u\left(x^{\star}\right)=t$. But then, by Lemma 4.11,

$$
\Lambda_{1}\left(\left|\nabla u\left(x^{\star}\right)\right|\right) \leq c_{u}-F\left(u\left(x^{\star}\right)\right)=0
$$

thence $\nabla u\left(x^{\star}\right)=0$, against the assumptions.

From now on, in the light of Lemma 4.13, we will suppose that

$$
c_{u}=F(m)
$$

since the case in which $c_{u}=F(M)$ may be treated similarly (simply, the rôles of $\bar{u}$ and $\underline{u}$ would switch in some arguments).

After the above analysis, we are now in a position to classify the behavior of the one-dimensional profiles at infinity:

Lemma 4.14. Let $u \in C^{1}\left(\mathbb{R}^{N}\right) \cap W^{1, \infty}\left(\mathbb{R}^{N}\right)$ be a stable weak solution of $(1.1)$ in $\mathbb{R}^{N}$, satisfying (4.12) and (4.13). Let $\underline{u}$ be as in (4.14) and suppose that it possesses one-dimensional symmetry, that is, there exist $\underline{\omega} \in S^{N-2}$ and $\underline{h}: \mathbb{R} \rightarrow \mathbb{R}$ in such a way that $\underline{u}\left(x^{\prime}\right)=\underline{h}\left(\underline{\omega} \cdot x^{\prime}\right)$, for any $x \in \mathbb{R}^{N-1}$.

Then, $\underline{h}$ satisfies one of the following:

A. $\underline{h}$ is constant,

B. $\left\{\underline{h}^{\prime}=0\right\}=\emptyset$,

C. There exists $\beta \in \mathbb{R}$ in such a way that $\underline{h}^{\prime}(t)<0$ for $t<\beta$ and $\underline{h}(t)=m$ for $t \geq \beta$.

D. There exists $\beta \in \mathbb{R}$ in such a way that $\underline{h}^{\prime}(t)>0$ for $t>\beta$ and $\underline{h}(t)=m$ for $t \leq \beta$.

E. There exist $\beta_{1} \leq \beta_{2} \in \mathbb{R}$ in such a way that $\underline{h}^{\prime}(t)<0$ for $t<\beta_{1}, \underline{h}^{\prime}(t)>0$ for $t>\beta_{2}$ and $\underline{h}(t)=m$ for $t \in\left[\beta_{1}, \beta_{2}\right]$.

Proof. Note that $\underline{h}(t)=\underline{u}(\underline{\omega} t)$, thence it satisfies (4.4), thanks to (4.16).

Also,

$$
\underline{h}(t)<M
$$

for any $t \in \mathbb{R}$, because if otherwise $\underline{h}(t)=M$ for some $t$, then

$$
M=\underline{h}(t)=\underline{u}(\underline{\omega} t)=\lim _{s \rightarrow-\infty} u(\underline{\omega} t, s) \leq u(\underline{\omega} t, 0) \leq M,
$$

due to (4.13), and so

$$
M=\max _{\mathbb{R}^{N}} u=u(\underline{\omega t}, 0),
$$

which implies $\nabla u(\underline{\omega t}, 0)=0$, in contradiction with (4.12). 
We then use the classification in Lemma 4.10: if $\underline{h}$ satisfies I or II there, then we are in cases A or B, and we are done.

We now show that case III of Lemma 4.10 is impossible in this circumstance. Indeed, if case III holds, by (4.10) and (4.26), we would have that $\underline{h}$ is strictly monotone in $\left(\beta_{1}, \beta_{2}\right)$ and

$$
F\left(\underline{h}\left(\beta_{1}\right)\right)=F\left(\underline{h}\left(\beta_{2}\right)=F(\inf \underline{h})=F(\underline{m})=F(m)\right.
$$

for any $t \in\left(\beta_{1}, \beta_{2}\right)$. But then, by (4.28), we see that

$$
F\left(\underline{h}\left(\beta_{1}\right)\right)=F\left(\underline{h}\left(\beta_{2}\right)\right)=c_{u}
$$

and so

$$
\left.\underline{h}\left(\beta_{1}\right), \underline{h}\left(\beta_{2}\right)\right) \in\{m, M\},
$$

thanks to Lemma 4.13.

Therefore, by (4.29), we get that $h\left(\beta_{1}\right)=h\left(\beta_{2}\right)=m$, but this is in contradiction with the fact that $h$ is strictly monotone in $\left(\beta_{1}, \beta_{2}\right)$.

Thus, the only remaining possibility is that $h$ satisfies IV of Lemma 4.10. Up to changing $t$ with $-t$, we may just consider the case in which the interval in IV of Lemma 4.10 is of the form $(-\infty, \beta)$. Then, by (4.11), (4.26) and (4.28),

$$
F(\underline{h}(\beta))=F(\underline{m})=F(m)=c_{u} .
$$

Thus, by Lemma 4.13, $h(\beta) \in\{m, M\}$. Therefore, by (4.29), $h(\beta)=m$.

Now, if $h(t)=m$ for $t \geq \beta$, then we are in case C. Thus, we may suppose that $\underline{h}\left(t^{\star}\right)>\bar{m}$ for some $t^{\star}>\beta$.

But then there must be $t^{\prime}>\beta$ in such a way that $\underline{h}^{\prime}\left(t^{\prime}\right)>0$. Hence, there exists an interval $\left(\beta_{2}, \beta_{3}\right)$, which we suppose as large as possible, with $\beta_{2}>\beta=$ : $\beta_{1}$ and $\beta_{3} \in\left(\beta_{2},+\infty\right) \cup\{+\infty\}$ in such a way that $h^{\prime}(t)>0$ for any $t \in\left(\beta_{2}, \beta_{3}\right)$. If $\beta_{3} \neq+\infty$, we would have that $\underline{h}^{\prime}\left(\beta_{2}\right)=\underline{h}^{\prime}\left(\beta_{3}\right)=0$ and, from (4.9), we would reduce to case III of Lemma 4.10 , that we have already shown to be impossible. Therefore, $\beta_{3}=+\infty$. Analogously, $h(t)$ must be equal to $m$ in $\left[\beta_{1}, \beta_{2}\right]$ because, if not there would be an interval $\left(\beta_{1}^{\prime}, \beta_{2}^{\prime}\right) \subset\left[\beta_{1}, \beta_{2}\right]$ in such a way that $\underline{h}^{\prime}(t) \neq 0$ in $\left(\beta_{1}^{\prime}, \beta_{2}^{\prime}\right)$ and $\underline{h}^{\prime}\left(\beta_{1}^{\prime}\right)=\underline{h}^{\prime}\left(\beta_{2}^{\prime}\right)=0$, reducing again to the impossible case III.

This shows that $\underline{h}^{\prime}(t) \neq 0$ for $t<\beta_{1}$ and $t>\beta_{2}$ and $\underline{h}(t)=m$ for $t \in\left[\beta_{1}, \beta_{2}\right]$, yielding case $\mathrm{E}$.

We remark that the possible occurrence of cases C, D and E in Lemma 4.14 is due to the possible degeneracy of our operator (non-degenerate operators do not admit non-constant solutions with plateaus, because of Comparison Principles). See Section 7.2 for explicit examples.

Corollary 4.15. The following global energy bound holds:

$$
\int_{-\infty}^{+\infty} \Lambda_{2}\left(\underline{h}^{\prime}(t)\right)-F\left(\underline{h}^{\prime}(t)\right)+c_{u} d t<+\infty .
$$


Proof. From (4.28), Lemma 4.2 and Corollary 4.9, we see that

$$
\int_{-\infty}^{+\infty} c_{u}-F\left(\underline{h}^{\prime}(t)\right) d t=\int_{-\infty}^{+\infty} \Lambda_{1}\left(\underline{h}^{\prime}(t)\right) d t \leq \mathrm{const} \int_{-\infty}^{+\infty} \Lambda_{2}\left(\underline{h}^{\prime}(t)\right) d t,
$$

hence the claim is proven if we show that

$$
\int_{-\infty}^{+\infty} \Lambda_{2}\left(\underline{h}^{\prime}(t)\right) d t<+\infty
$$

To this extent, we consider the different cases in Lemma 4.14 and we make use of Lemma 4.4.

Namely, in case A, the quantity in (4.31) vanishes and we are done.

In case $\mathrm{B}$,

$$
\begin{aligned}
\int_{-\infty}^{+\infty} \Lambda_{2}\left(\underline{h}^{\prime}(t)\right) d t & =\lim _{\substack{\alpha \rightarrow-\infty \\
\beta \rightarrow+\infty}} \int_{\alpha}^{\beta} \Lambda_{2}\left(\underline{h}^{\prime}(t)\right) d t \\
& \leq \lim _{\substack{\alpha \rightarrow-\infty \\
\beta \rightarrow+\infty}} \operatorname{const}|\underline{h}(\beta)-\underline{h}(\alpha)| \\
& \leq 2 \text { const } M .
\end{aligned}
$$

In case $\mathrm{C}$,

$$
\begin{aligned}
\int_{-\infty}^{+\infty} \Lambda_{2}\left(\underline{h}^{\prime}(t)\right) d t & =\lim _{\alpha \rightarrow-\infty} \int_{\alpha}^{\beta} \Lambda_{2}\left(\underline{h}^{\prime}(t)\right) d t \\
& \leq \lim _{\alpha \rightarrow-\infty} \operatorname{const}|\underline{h}(\beta)-\underline{h}(\alpha)| \\
& \leq 2 \text { const } M,
\end{aligned}
$$

and case $\mathrm{D}$ is analogous.

Finally, in case E,

$$
\begin{aligned}
\int_{-\infty}^{+\infty} \Lambda_{2}\left(\underline{h}^{\prime}(t)\right) d t & =\lim _{\substack{\alpha \rightarrow-\infty \\
\beta \rightarrow+\infty}} \int_{\alpha}^{\beta_{1}} \Lambda_{2}\left(\underline{h}^{\prime}(t)\right) d t+\int_{\beta_{2}}^{\beta} \Lambda_{2}\left(\underline{h}^{\prime}(t)\right) d t \\
& \leq \lim _{\substack{\alpha \rightarrow-\infty \\
\beta \rightarrow+\infty}} \operatorname{const}\left(\left|\underline{h}\left(\beta_{1}\right)-\underline{h}(\alpha)\right|+\left|\underline{h}(\beta)-\underline{h}\left(\beta_{2}\right)\right|\right) \\
& \leq 4 \operatorname{const} M,
\end{aligned}
$$

which completes the proof of the desired result.

The above global energy estimates imply that $u$ has "the good energy" in balls: 
Corollary 4.16. Let $u \in C^{1}\left(\mathbb{R}^{N}\right) \cap W^{1, \infty}\left(\mathbb{R}^{N}\right)$ be a stable weak solution of $(1.1)$ in $\mathbb{R}^{N}$, satisfying (4.12) and (4.13). Let $\underline{u}$ possess one-dimensional symmetry. Then,

$$
\int_{B_{R}} \Lambda_{2}(|\nabla u(x)|)-F(u(x))+c_{u} d x \leq K R^{N-1},
$$

for a suitable $K \geq 1$.

Proof. By (4.30), we have that

$$
\begin{aligned}
& \int_{B_{R}} \Lambda_{2}\left(\left|\nabla \underline{u}\left(x^{\prime}\right)\right|\right)-F\left(\underline{u}\left(x^{\prime}\right)\right)+c_{u} d x \\
& =\int_{B_{R}} \Lambda_{2}\left(\underline{h^{\prime}}\left(\underline{\omega} \cdot x^{\prime}\right)\right)-F\left(\underline{h}\left(\underline{\omega} \cdot x^{\prime}\right)\right)+c_{u} d x \\
& =\int_{B_{R}} \Lambda_{2}\left(\underline{h^{\prime}}\left(\underline{\omega} \cdot x^{\prime}\right)\right)-F\left(\underline{h}\left(\underline{\omega} \cdot x^{\prime}\right)\right)+c_{u} d x^{\prime} d x_{N} \\
& =\int_{\left(y^{\prime \prime}, t, x_{N}\right) \in\left(\mathbb{R}^{N-2} \times \mathbb{R} \times \mathbb{R}\right) \cap B_{R}} \Lambda_{2}\left(\underline{h}^{\prime}(t)\right)-F(\underline{h}(t))+c_{u} d y^{\prime \prime} d t d x_{N} \\
& \leq \text { const } R^{N-1} .
\end{aligned}
$$

Also, recalling Lemma 4.1 and (4.15),

$$
\begin{aligned}
& \int_{B_{R}} \Lambda_{2}(|\nabla u(x)|)-F(u(x))+c_{u} d x \\
& =E_{R}(u)+c_{u}\left|B_{R}\right| \\
& \leq \lim _{t \rightarrow+\infty} E_{R}\left(u^{t}\right)+c_{u}\left|B_{R}\right|+\text { const } R^{N-1} \\
& =\int_{B_{R}} \Lambda_{2}\left(\left|\nabla \underline{u}\left(x^{\prime}\right)\right|\right)-F\left(\underline{u}\left(x^{\prime}\right)\right)+c_{u} d x+\text { const } R^{N-1} .
\end{aligned}
$$

The latter estimate and (4.32) imply the desired claim.

\section{First proof of Theorem 1.2, via a capacity argument}

We consider the graph of a function $v \in C^{1}\left(\mathbb{R}^{N}\right)$ as a surface in $\mathbb{R}^{N+1}$ and we set

$$
Y=Y(x):=(x, v(x)) \in \mathbb{R}^{N} \times \mathbb{R} .
$$

We will derive suitable capacity estimates on the graph of $v$ under the assumption that

$$
\int_{|Y(x)| \leq \rho} a(|\nabla v(x)|)|\nabla v(x)|^{2} d x \leq C \rho^{2},
$$

as long as $\rho \geq C$, for a suitable $C>0$. 
The advantage of such estimates is that the capacity is evaluated on the graph of $v$, not on the whole space, and this makes it possible to obtain two-dimensional capacity estimates even in $\mathbb{R}^{3}$, as we will see below.

The main technical estimate needed is the following:

Lemma 5.1. Suppose that $v \in C^{1}\left(\mathbb{R}^{N}\right)$ satisfies (5.1).

Then, there exists $C \geq 1$ in such a way that

$$
\int_{\sqrt{\rho} \leq|Y| \leq \rho} \frac{a(|\nabla v|)|\nabla v|^{2}}{|Y|^{2}} d x \leq C \ln \rho,
$$

as long as $\rho \geq C$.

Proof. This argument is a modification of the classical ones on [24, page 24] and [18, page 403].

Since

$$
2 \int_{|Y|}^{\rho} \tau^{-3} d \tau=|Y|^{-2}-\rho^{-2},
$$

we obtain, from Fubini's theorem, that

$$
\begin{aligned}
& \int_{\sqrt{\rho} \leq|Y| \leq \rho} a(|\nabla v|)|\nabla v|^{2}\left(|Y|^{-2}-\rho^{-2}\right) d x \\
& =2 \int_{\sqrt{\rho} \leq|Y| \leq \rho}\left(\int_{|Y|}^{\rho} a(|\nabla v(x)|)|\nabla v(x)|^{2} \tau^{-3} d \tau\right) d x \\
& =2 \int_{\sqrt{\rho}}^{\rho}\left(\int_{\sqrt{\rho} \leq|Y| \leq \tau} a(|\nabla v(x)|)|\nabla v(x)|^{2} \tau^{-3} d x\right) d \tau \\
& =2 \int_{\sqrt{\rho}}^{\rho} \tau^{-3}\left(\int_{\sqrt{\rho} \leq|Y| \leq \tau} a(|\nabla v(x)|)|\nabla v(x)|^{2} d x\right) d \tau .
\end{aligned}
$$

Accordingly, from (5.1),

$$
\begin{aligned}
& \int_{\sqrt{\rho} \leq|Y| \leq \rho} a(|\nabla v|)|\nabla v|^{2}\left(|Y|^{-2}-\rho^{-2}\right) d x \\
& \leq \text { const } \int_{\sqrt{\rho}}^{\rho} \tau^{-1} d \tau \leq \text { const } \ln \rho,
\end{aligned}
$$

if $\rho$ is large enough. 
Using this and (5.1) once more, we conclude that

$$
\begin{aligned}
& \int_{\sqrt{\rho} \leq|Y| \leq \rho} a(|\nabla v|)|\nabla v|^{2}|Y|^{-2} d x \\
& =\rho^{-2} \int_{\sqrt{\rho} \leq|Y| \leq \rho} a(|\nabla v|)|\nabla v|^{2} d x \\
& \quad+\int_{\sqrt{\rho} \leq|Y| \leq \rho} a(|\nabla v|)|\nabla v|^{2}\left(|Y|^{-2}-\rho^{-2}\right) d x \\
& \leq \text { const }+ \text { const } \ln \rho,
\end{aligned}
$$

as desired.

The hypotheses of next result need to be quite general, since we apply it in the proofs of both Theorems 1.2 and 1.4.

Lemma 5.2. Let (1.2), (1.3) hold.

Suppose that $v \in C^{1}\left(\mathbb{R}^{N}\right) \cap C^{2}(\{\nabla v \neq 0\})$ with $\nabla v \in W_{\text {loc }}^{1,2}\left(\mathbb{R}^{N}\right)$ is a stable weak solution of (1.1) and that it satisfies (5.1).

Assume that

$$
|\nabla v(x)|^{2} \lambda_{1}(|\nabla v(x)|) \leq C a(|\nabla v(x)|),
$$

for any $x \in \mathbb{R}^{N}$ as long as $|\nabla v(x)| \geq C^{\prime}$, for suitable $C, C^{\prime}>0$.

Suppose that either (A1) or (A2) holds.

Assume also that either $N=2$ or that

$$
\lambda_{1}(t) \leq C^{\prime \prime} a(t),
$$

for any $t \in\left(0, C^{\prime}\right]$, for a suitable $C^{\prime \prime}>0$.

Then, $v$ possesses one-dimensional symmetry, in the sense that there exists $\bar{v}$ : $\mathbb{R} \rightarrow \mathbb{R}$ and $\omega \in S^{N-1}$ in such a way that $v(x)=\bar{v}(\omega \cdot x)$, for any $x \in \mathbb{R}^{N}$.

Proof. We first notice that, if $|\nabla v(x)| \geq C^{\prime}$, then

$$
\begin{aligned}
|\nabla v(x)|^{2} \lambda_{1}(|\nabla v(x)|) & \leq \frac{C}{\left(C^{\prime}\right)^{2}}\left(C^{\prime}\right)^{2} a(|\nabla v(x)|) \\
& \leq \frac{C}{\left(C^{\prime}\right)^{2}}|\nabla v(x)|^{2} a(|\nabla v(x)|),
\end{aligned}
$$

thanks to (5.3).

We now define

$$
\Xi:= \begin{cases}1 & \text { if } N=2, \\ 0 & \text { if } N \geq 3\end{cases}
$$


We claim that

$$
|\nabla v(x)|^{2} \lambda_{1}(|\nabla v(x)|) \leq \text { const }\left(\Xi+|\nabla v(x)|^{2} a(|\nabla v(x)|)\right)
$$

for any $x \in \mathbb{R}^{N}$.

To prove (5.7) we distinguish the cases $N=2$ and $N \geq 3$.

If $N \geq 3$, we have that (5.4) is satisfied. We exploit this and (5.5) to conclude that

$$
|\nabla v(x)|^{2} \lambda_{1}(|\nabla v(x)|) \leq C^{\prime \prime \prime}|\nabla v(x)|^{2} a(|\nabla v(x)|) \quad \text { for any } x \in \mathbb{R}^{N},
$$

where

$$
C^{\prime \prime \prime}:=\frac{C}{\left(C^{\prime}\right)^{2}}+C^{\prime \prime} .
$$

This proves (5.7) when $N=3$.

We now prove (5.7) when $N=2$. To this extent, we observe that $\lambda_{1} \in$ $L_{\mathrm{loc}}^{\infty}([0,+\infty))$, because of either (A1) or (A2), thence

$$
t^{2} \lambda_{1}(t) \leq \mathrm{const}
$$

if $t \in\left[0, C^{\prime}\right]$.

This and (5.5) imply that

$$
|\nabla v(x)|^{2} \lambda_{1}(|\nabla v(x)|) \leq \mathrm{const}\left(1+|\nabla v(x)|^{2} a(|\nabla v(x)|)\right)
$$

for any $x \in \mathbb{R}^{N}$, hence (5.7) holds when $N=2$ too.

This completes the proof of (5.7).

Now, we use (5.3) and (5.7) to conclude that

$$
|\nabla v(x)|^{4} \lambda_{1}(|\nabla v(x)|) \leq \mathrm{const}\left(\Xi+|\nabla v(x)|^{2} a(|\nabla v(x)|)\right)
$$

for any $x \in \mathbb{R}^{N}$.

Also, since, obviously, (5.7) holds with $\lambda_{1}$ replaced by $\lambda_{2}$, due to (1.5), we deduce, recalling Lemma 2.1, that

$$
|\nabla v(x)|^{2}|A(\nabla v(x))| \leq \mathrm{const}\left(\Xi+|\nabla v(x)|^{2} a(|\nabla v(x)|)\right)
$$

for any $x \in \mathbb{R}^{N}$.

Also, by Lemma 5.1 and (5.6),

$$
\int_{B_{R} \backslash B_{\sqrt{R}}} \frac{\Xi+|\nabla v(x)|^{2} a(|\nabla v|)}{|Y|^{2}} d x \leq C \ln R,
$$

as long as $R$ is large enough. 
Then, given $R>0$ (to be taken appropriately large in what follows) and $x \in$ $\mathbb{R}^{N}$, we now define

$$
\varphi_{R}(x):= \begin{cases}1 & \text { if }|Y| \leq \sqrt{R}, \\ \frac{2 \ln (R /|Y|)}{\ln R} & \text { if } \sqrt{R}<|Y|<R, \\ 0 & \text { if }|Y| \geq R .\end{cases}
$$

By construction, $\varphi_{R}$ is a Lipschitz function and

$$
\nabla \varphi_{R}(x)=-\frac{\operatorname{const}(x+v(x) \nabla v(x))}{|Y|^{2} \ln R}
$$

for any $x \in \mathbb{R}^{N}$ such that $\sqrt{R}<|Y|<R$.

Consequently, from (2.2),

$$
\begin{aligned}
& \left(A(\nabla v(x)) \nabla \varphi_{R}(x)\right) \cdot \nabla \varphi_{R}(x) \\
& \leq \frac{\text { const }}{|Y|^{4} \ln ^{2} R}\left[(A(\nabla v(x)) x) \cdot x+v^{2}(x)(A(\nabla v(x)) \nabla v(x)) \cdot \nabla v(x)\right] .
\end{aligned}
$$

Additionally, by (5.11),

$$
\begin{aligned}
|\nabla v(x)|^{2}(A(\nabla v(x)) x) \cdot x & \leq \text { const }\left(\Xi+|\nabla v(x)|^{2} a(|\nabla v(x)|)\right)|x|^{2} \\
& \leq \operatorname{const}\left(\Xi+|\nabla v(x)|^{2} a(|\nabla v(x)|)\right)|Y|^{2},
\end{aligned}
$$

and, by (2.1) and (5.10),

$$
\begin{aligned}
& v^{2}(x)|\nabla v(x)|^{2}(A(\nabla v(x)) \nabla v(x)) \cdot \nabla v(x) \\
& =v^{2}(x)|\nabla v(x)|^{4} \lambda_{1}(|\nabla v(x)|) \\
& \leq \text { const }\left(\Xi+|\nabla v(x)|^{2} a(|\nabla v(x)|)\right)|Y|^{2} .
\end{aligned}
$$

So (5.13), (5.14) and (5.15) imply

$$
\begin{aligned}
& |\nabla v(x)|^{2}\left(A(\nabla v(x)) \nabla \varphi_{R}(x)\right) \cdot \nabla \varphi_{R}(x) \\
& \leq \frac{\operatorname{const}\left(\Xi+|\nabla v(x)|^{2} a(|\nabla v(x)|)\right)}{|Y|^{2} \ln ^{2} R} .
\end{aligned}
$$


Thus, exploiting again Theorem 2.5,

$$
\begin{aligned}
& \int_{\{\nabla v \neq 0\} \cap B_{\sqrt{R}}}\left[\lambda_{1}(|\nabla v|)\left|\nabla_{L_{v, x}}\right| \nabla v||^{2}+\lambda_{2}(|\nabla v|)|\nabla v|^{2} \kappa_{1}^{2}\right] d x \\
& \leq \int_{\mathbb{R}^{2}}|\nabla v(x)|^{2}\left(A(\nabla v(x)) \nabla \varphi_{R}(x)\right) \cdot \nabla \varphi_{R}(x) d x \\
& \leq \int_{B_{R} \backslash B_{\sqrt{R}}} \frac{\operatorname{const}\left(\Xi+|\nabla v(x)|^{2} a(|\nabla v(x)|)\right)}{|Y|^{2} \ln ^{2} R} d x .
\end{aligned}
$$

Therefore, by (5.12), if $R$ is conveniently large,

$$
\begin{aligned}
& \int_{\{\nabla v \neq 0\} \cap B_{\sqrt{R}}}\left[\lambda_{1}(|\nabla v|)\left|\nabla_{L_{v, x}}\right| \nabla v||^{2}+\lambda_{2}(|\nabla v|)|\nabla v|^{2} \kappa_{1}^{2}\right] d x \\
& \leq \frac{\text { const } \ln R}{\ln ^{2} R} .
\end{aligned}
$$

By taking $R$ arbitrarily large, we thus conclude that

$$
\int_{\{\nabla v \neq 0\}}\left[\lambda_{1}(|\nabla v|)\left|\nabla_{L_{v, x}}\right| \nabla v||^{2}+\lambda_{2}(|\nabla v|)|\nabla v|^{2} \kappa_{1}^{2}\right] d x=0
$$

and so, by Lemma 2.1, that $\nabla_{L_{v, x}}|\nabla v|(x)=0=\kappa_{1}(x)$ at any $x \in\{\nabla v \neq 0\}$.

Consequently, by Lemma 2.11, $v$ possesses one-dimensional symmetry.

\subsection{Completion of the first proof of Theorem 1.2}

We observe that $\underline{u}$ and $\bar{u}$ are, in this case, functions on $\mathbb{R}^{2}$. Hence, by (4.17) and Corollary 2.6, we have that

$$
\kappa_{1, \underline{u}}=\kappa_{1, \bar{u}}=0 \text { and } \nabla_{L_{\underline{u}, x^{\prime}}}|\nabla \underline{u}|=\nabla_{L_{\bar{u}, x^{\prime}}}|\nabla \bar{u}| .
$$

Therefore, by Lemma 2.11, both $\underline{u}$ and $\bar{u}$ possess one-dimensional symmetry and so, by (4.27) and Corollary 4.16,

$$
\int_{B_{R}} \Lambda_{2}(|\nabla u(x)|) d x \leq \int_{B_{R}} \Lambda_{2}(|\nabla u(x)|)-F(u(x))+c_{u} d x \leq \text { const } R^{2} .
$$

Also, if either (B1) or (B2) holds, we have that (1.2) and (1.3) are satisfied, due to Lemma 2.1.

Moreover, recalling also Lemma 4.2, we see that (5.4) is fulfilled and (5.3) is empty, because $u$ has bounded gradient. 
Thus, if $u$ is as requested in Theorem 1.2, we exploit Corollaries 4.3 and 4.16 to get that

$$
\begin{aligned}
& \int_{|(x, u(x))| \leq R} a(|\nabla u(x)|)|\nabla u(x)|^{2} d x \\
& \leq \int_{B_{R}} a(|\nabla u(x)|)|\nabla u(x)|^{2} d x \\
& \leq \mathrm{const} \int_{B_{R}} \Lambda_{2}(|\nabla u(x)|) d x \\
& \leq \mathrm{const} \int_{B_{R}} \Lambda_{2}(|\nabla u(x)|)-F(u(x))+c_{u} d x \\
& \leq \text { const } R^{2},
\end{aligned}
$$

hence (5.1) is fulfilled by $u$.

The above observations give that the assumptions of Lemma 5.2 are fulfilled, thence, by such a result, $u$ possesses one-dimensional symmetry, thus completing the first proof of Theorem 1.2.

\section{Second proof of Theorem 1.2, via a Liouville type argument}

\subsection{A Liouville type result}

We now point out a Liouville type result, which is a variation of the one in $[3,17]$ and it is closely related to analogous estimates performed in [5,7].

Lemma 6.1. Let $B \in L^{\infty}\left(\mathbb{R}^{N}\right.$, Mat $\left.(N \times N)\right)$ be such that $B(x)$ is symmetric and positive definite for any $x \in \mathbb{R}^{N}$. Let $\omega \in L^{\infty}\left(\mathbb{R}^{N}\right)$, with $\omega(x)>0$ for any $x \in \mathbb{R}^{N}$. Suppose that $\zeta \in C^{1}\left(\mathbb{R}^{N}\right)$ is a weak solution of

$$
\operatorname{div}(\omega(x) B(x) \nabla \zeta(x))=0 .
$$

Assume also that there exist $C>0$ in such a way that

$$
\int_{\mathbb{R}^{N}} \omega(x) \zeta^{2}(x)(B(x) \tau(x)) \cdot \tau(x) d x \leq C\|\tau\|_{L^{\infty}\left(\mathbb{R}^{N}\right)} R^{2},
$$

for any $\tau \in C_{0}^{\infty}\left(\mathbb{R}^{N}, \mathbb{R}^{N}\right)$ supported in $B_{2 R}$.

Then, $\zeta$ is constant.

Proof. The proof is a Caccioppoli type argument modified from [3]. We take $\alpha \in$ $C_{0}^{\infty}\left(B_{2}\right)$ so that $0 \leq \alpha(x) \leq 1$ for any $x \in \mathbb{R}^{N}$ and $\alpha(x)=1$ for any $x \in B_{1}$. We also set $\alpha_{R}(x):=\alpha(x / R), \tau_{R}(x):=\nabla \alpha(x / R)$ and $\phi_{R}(x):=\left(\alpha_{R}(x)\right)^{2} \zeta(x)$. 
From (6.1),

$$
\begin{aligned}
& \int_{\mathbb{R}^{N}} \alpha_{R}^{2} \omega(B \nabla \zeta) \cdot \nabla \zeta d x \\
& =\int_{\mathbb{R}^{N}} \omega(B \nabla \zeta) \cdot \nabla \phi_{R} d x-2 \int_{\mathbb{R}^{N}} \alpha_{R} \zeta \omega(B \nabla \zeta) \cdot \nabla \alpha_{R} d x \\
& \leq 0+2 \int_{R \leq|x| \leq 2 R} \alpha_{R}|\zeta| \omega\left|(B \nabla \zeta) \cdot \nabla \alpha_{R}\right| d x
\end{aligned}
$$

We now fix an auxiliary parameter $\delta>0$, and we exploit (2.2) with $V:=\sqrt{\delta \omega} \alpha_{R} \nabla \zeta$ and $W:=\sqrt{\delta^{-1} \omega}|\zeta| \nabla \alpha_{R}$, so we conclude that

$$
2 \alpha_{R}|\zeta| \omega\left|(B \nabla \zeta) \cdot \nabla \alpha_{R}\right| \leq \delta \alpha_{R}^{2} \omega(B \nabla \zeta) \cdot \nabla \zeta+\delta^{-1} \omega \zeta^{2}\left(B \nabla \alpha_{R}\right) \cdot \nabla \alpha_{R}
$$

and therefore

$$
\begin{aligned}
& \int_{\mathbb{R}^{N}} \alpha_{R}^{2} \omega(B \nabla \zeta) \cdot \nabla \zeta d x \\
& \leq \delta \int_{R \leq|x| \leq 2 R} \alpha_{R}^{2} \omega(B \nabla \zeta) \cdot \nabla \zeta d x \\
& \quad+\delta^{-1} \int_{R \leq|x| \leq 2 R} \omega \zeta^{2}\left(B \nabla \alpha_{R}\right) \cdot \nabla \alpha_{R} d x
\end{aligned}
$$

Note also that, from (6.2),

$$
\int_{R \leq|x| \leq 2 R} \omega \zeta^{2}\left(B \nabla \alpha_{R}\right) \cdot \nabla \alpha_{R} d x=R^{-2} \int_{\mathbb{R}^{N}} \omega \zeta^{2}\left(B \nabla \tau_{R}\right) \cdot \nabla \tau_{R} d x \leq C^{\prime}
$$

for a suitable $C^{\prime}>0$.

Accordingly, if $\delta<1$, from (6.3),

$$
\begin{aligned}
& (1-\delta) \int_{B_{R}} \omega(B \nabla \zeta) \cdot \nabla \zeta d x \\
& \leq(1-\delta) \int_{\mathbb{R}^{N}} \alpha_{R}^{2} \omega(B \nabla \zeta) \cdot \nabla \zeta d x \\
& \leq C^{\prime} \delta^{-1}
\end{aligned}
$$

By sending $R \rightarrow+\infty$, we thus obtain that

$$
\int_{\mathbb{R}^{N}} \omega(B \nabla \zeta) \cdot \nabla \zeta d x<+\infty
$$


and therefore

$$
\lim _{R \rightarrow+\infty} \int_{|x| \geq R} \omega(B \nabla \zeta) \cdot \nabla \zeta d x=0 .
$$

Using this and sending $R \rightarrow+\infty$ in (6.3), we conclude that

$$
\begin{aligned}
& \int_{\mathbb{R}^{N}} \omega(B \nabla \zeta) \cdot \nabla \zeta d x \\
& \leq \delta \lim _{R \rightarrow+\infty} \int_{R \leq|x| \leq 2 R} \omega(B \nabla \zeta) \cdot \nabla \zeta d x \\
& \quad+\delta^{-1} \lim _{R \rightarrow+\infty} \int_{R \leq|x| \leq 2 R} \omega \zeta^{2}\left(B \nabla \alpha_{R}\right) \cdot \nabla \alpha_{R} d x \\
& \leq 0+C^{\prime} \delta^{-1} .
\end{aligned}
$$

By sending now $\delta \rightarrow+\infty$, we conclude that

$$
\int_{\mathbb{R}^{N}} \omega(B \nabla \zeta) \cdot \nabla \zeta d x=0
$$

and so that $\nabla \zeta=0$.

The relation between (6.1) and our problem is given by the following standard observation (compare, e.g., with $[3,7]$ ):

Lemma 6.2. Let $u \in C^{2}\left(\mathbb{R}^{N}\right)$ be a solution of (1.1), such that $|\nabla u| \in L^{\infty}\left(\mathbb{R}^{N}\right)$ and $\partial_{N} u(x)>0$ for any $x \in \mathbb{R}^{N}$.

Fix $j \in\{1, \ldots, N-1\}$ and define $\zeta(x):=u_{j}(x) / u_{N}(x), \omega(x):=\left(u_{N}(x)\right)^{2}$ and $B(x):=A(\nabla u(x))$.

Then, $B \in L^{\infty}\left(\mathbb{R}^{N}, \operatorname{Mat}(N \times N)\right), B(x)$ is symmetric and positive definite for any $x \in \mathbb{R}^{N}, \omega \in L^{\infty}\left(\mathbb{R}^{N}\right), \zeta \in C^{1}\left(\mathbb{R}^{N}\right)$ and it is a weak solution of (6.1).

Proof. The regularity of $B, \omega$ and $\zeta$ is obvious and Lemma 2.1 gives that $B(x)$ is positive definite.

Also,

$$
\omega \zeta_{i}=u_{N} u_{i j}-u_{j} u_{i N}
$$

and so, by Lemma 2.2,

$$
\begin{aligned}
\operatorname{div}(\omega B \nabla \zeta)= & \operatorname{div}\left(u_{N} B \nabla u_{j}-u_{j} B \nabla u_{N}\right) \\
& =u_{N} \operatorname{div}\left(B \nabla u_{j}\right)+\left(B \nabla u_{j}\right) \cdot \nabla u_{N}-\operatorname{div}\left(u_{j} B \nabla u_{N}\right) \\
& =u_{N} \operatorname{div}\left(B \nabla u_{j}\right)-u_{j} \operatorname{div}\left(B \nabla u_{N}\right) \\
& =-u_{N} f^{\prime}(u) u_{j}+u_{j} f^{\prime}(u) u_{N}=0
\end{aligned}
$$

as claimed. 

bound:

The symmetry property in our case is thus a consequence of a "kinetic energy"

Corollary 6.3. Let $u \in C^{2}\left(\mathbb{R}^{N}\right)$ be a solution of (1.1), such that $|\nabla u| \in L^{\infty}\left(\mathbb{R}^{N}\right)$ and $\partial_{N} u(x)>0$ for any $x \in \mathbb{R}^{N}$.

Suppose that

$$
\int_{B_{R}} \Lambda_{2}(|\nabla u(x)|) d x \leq C R^{2},
$$

for any $R>0$, for a suitable $C>0$.

Then, $u$ possesses one-dimensional symmetry.

Proof. If $\xi, v \in \mathbb{R}^{N}$ with $|v| \leq 1$ and $|\xi| \leq M$, then

$$
|\xi|^{2}(A(\xi) v) \cdot v \leq C_{M} \Lambda_{2}(|\xi|),
$$

for a suitable $C_{M}>0$, because of Lemma 4.2 and Corollary 4.3.

We now take $B, \omega$ and $\zeta$ as in Lemma 6.2 and we make use of (6.4) and (6.5) to deduce that

$$
\begin{aligned}
& \int_{\mathbb{R}^{N}} \omega(x) \zeta^{2}(x)(B(x) \tau(x)) \cdot \tau(x) d x \\
& \leq \int_{\mathbb{R}^{N}}|\nabla u(x)|^{2}(A(\nabla u(x)) \tau(x)) \cdot \tau(x) d x \\
& \leq \text { const }\|\tau\|_{L^{\infty}\left(\mathbb{R}^{N}\right)} \int_{B_{2 R}} \Lambda_{2}(|\nabla u(x)|) d x \\
& \leq \text { const }\|\tau\|_{L^{\infty}\left(\mathbb{R}^{N}\right)} R^{2},
\end{aligned}
$$

for any $\tau \in C_{0}^{\infty}\left(\mathbb{R}^{N}, \mathbb{R}^{N}\right)$ supported in $B_{2 R}$.

This shows that (6.2) holds and so, from Lemma 6.1, we get that $u_{j}=c_{j} u_{N}$, for a suitable $c_{j} \in \mathbb{R}$, for any $j=1, \ldots, N-1$.

Hence, if $\varpi:=\left(c_{1}, \ldots, c_{N-1}, 1\right)$, we have that $\partial_{v} u$ is identically zero for any $v$ orthogonal to $\varpi$, which yields the desired result.

\subsection{Completion of the second proof of Theorem 1.2}

The second proof of Theorem 1.2 is ended thanks to (5.16) and Corollary 6.3.

\section{Stability criteria}

\subsection{Monotonicity and stability}

It is a standard fact of Allen-Cahn type equations that solutions that are strictly monotone in one variable are stable. We provide a generalization of this fact in our setting: 
Lemma 7.1. Let $u \in C^{1}\left(\mathbb{R}^{N}\right) \cap C^{2}(\{\nabla u \neq 0\})$, with $\nabla u \in W_{\mathrm{loc}}^{1,2}\left(\mathbb{R}^{N}\right)$ be a weak solution of (1.1). Suppose that either (A2) holds or that $\{\nabla u=0\}=\emptyset$. Assume that $\partial_{x_{N}} u \geq 0$.

Then,

$$
\int_{\mathbb{R}^{N}}(A(\nabla u) \nabla \phi) \cdot \nabla \phi d x-\int_{\left\{\partial_{x_{N}} u>0\right\}} f^{\prime}(u) \phi^{2} d x \geq 0
$$

for any smooth and compactly supported function $\phi$.

In particular, if $u \in C^{2}\left(\mathbb{R}^{N}\right)$ is a solution of $(1.1)$ in $\mathbb{R}^{N}$ with $\partial_{x_{N}} u>0$, then it is stable.

Proof. Let $u_{N}:=\partial_{x_{N}} u$ and fix $\epsilon>0$ (in fact, if $\partial_{x_{N}} u>0$, one can simply take $\epsilon=0$ in what follows and the argument slightly simplifies).

Let $\phi$ be a smooth, compactly supported function and $\psi:=\phi^{2} /\left(u_{N}+\epsilon\right)$. We write $A:=A(\nabla u)$ and we use (2.2) with $V:=\left(\phi \nabla u_{N}\right) /\left(u_{N}+\epsilon\right)$ and $W:=\nabla \phi$ to obtain that

$$
\frac{2 \phi}{u_{N}+\epsilon} A \nabla u_{N} \cdot \nabla \phi-\frac{\phi^{2}}{\left(u_{N}+\epsilon\right)^{2}} A \nabla u_{N} \cdot \nabla u_{N} \leq A \nabla \phi \cdot \nabla \phi .
$$

From this and Lemma 2.4,

$$
\begin{aligned}
0 & =\int A \nabla u_{N} \cdot \nabla \psi-f^{\prime}(u) u_{N} \psi d x \\
& =\int \frac{2 \phi}{u_{N}+\epsilon} A \nabla u_{N} \cdot \nabla \phi-\frac{\phi^{2}}{\left(u_{N}+\epsilon\right)^{2}} A \nabla u_{N} \cdot \nabla u_{N}-f^{\prime}(u) \phi^{2} \frac{u_{N}}{u_{N}+\epsilon} d x \\
& \leq \int A \nabla \phi \cdot \nabla \phi-f^{\prime}(u) \phi^{2} \frac{u_{N}}{u_{N}+\epsilon} d x .
\end{aligned}
$$

By taking $\epsilon$ arbitrarily small, we obtain (7.1).

We point out that $\left\{\partial_{x_{N}} u>0\right\} \cap \mathcal{N}_{u}$ has measure zero, due to (2.12). This also implies that $u$ is stable if $\partial_{x_{N}} u>0$.

\subsection{Degenerate examples}

Our scope is now to show by examples that interesting degenerate cases are covered by our setting. This part is not used in the proofs of the main results, and it may thus be skipped by the uninterested reader.

Proposition 7.2. Let $p>2$. Then, there exist $w \in C^{2}\left(\mathbb{R}^{N}\right)$ and $f \in C^{1}(\mathbb{R})$ in such a way that

- $w$ is a stable solution of $\Delta_{p} w+f(w)=0$ having one-dimensional symmetry,

- $0 \leq w(x) \leq 1$ and $\partial_{x_{N}} w(x) \geq 0$ for any $x \in \mathbb{R}^{N}$,

- $w(x)=0$ if $x_{N} \leq 0$ and $w(x)=1$ if $x_{N} \geq 1$. 
Proof. Given $p>2$, we fix $\alpha$ such that

$$
0<\alpha<\min \left\{\frac{1}{2}, \frac{p-2}{p}\right\} .
$$

We define

$$
W(t):= \begin{cases}0 & \text { if } t \leq 0, \\ 1-\left(1-t^{1 / \alpha}\right)^{1 / \alpha} & \text { if } 0<t<1 \\ 1 & \text { if } t \geq 1\end{cases}
$$

and $w(x)=w\left(x_{1}, \ldots, x_{N}\right):=W\left(x_{N}\right)$. Since $1 / \alpha>2$, we have that $w \in C^{2}\left(\mathbb{R}^{N}\right)$, and clearly $\partial_{x_{N}} w \geq 0$.

A straightforward calculation shows that

$$
\Delta_{p} w+f(w)=0
$$

where

$$
f(r):=\frac{\kappa+\alpha}{\alpha^{2 p}} \cdot\left((1-r)\left(1-(1-r)^{\alpha}\right)\right)^{\kappa} \cdot\left(1-(\alpha+1)(1-r)^{\alpha}\right) \chi_{(0,1)}(r)
$$

and

$$
\kappa:=p-\alpha p-1 .
$$

Note that $\kappa>1$, thence $f \in C^{1}(\mathbb{R})$.

Moreover,

$$
\left\{\partial_{x_{N}} w=0\right\}=\{w=0\} \cup\{w=1\}
$$

and so $f^{\prime}(w(x))=0$ for any $x \in\left\{\partial_{x_{N}} w=0\right\}$. Consequently,

$$
\int_{\partial_{x_{N}} w>0} f^{\prime}(w) \phi^{2} d x=\int_{\mathbb{R}^{N}} f^{\prime}(w) \phi^{2} d x
$$

for any smooth and compactly supported $\phi$.

Accordingly, $w$ is stable, due to (7.1).

The example given in Proposition 7.2 shows that conditions (A1) and (A2) do not coincide in general: indeed, of course, the $p$-Laplacian for $1<p<2$ satisfies (A1) but not (A2), and the example given in Proposition 7.2 shows that there are cases on which Theorem 1.1 applies that are covered by (A2) and not by (A1), due to plateaus.

Case III in Lemma 4.10 is also embodied by the example of Proposition 7.2.

Proposition 7.3. Let $p>2$. Then, there exist $w \in C^{2}\left(\mathbb{R}^{N}\right)$ and $f \in C^{1}(\mathbb{R})$ in such a way that

- $w$ is a stable solution of $\Delta_{p} w+f(w)=0$ having one-dimensional symmetry, 
- $0 \leq w(x) \leq 1$ and $\partial_{x_{N}} w(x) \geq 0$ for any $x \in \mathbb{R}^{N}$,

- $w(x)=0$ if $x_{N} \leq 0, w(x)>0$ if $x_{N}>0$ and

$$
\lim _{x_{N} \rightarrow+\infty} w(x)=1 .
$$

Proof. Given $p>2$, we fix $\alpha$ as in (7.2) and we define

$$
W(t):= \begin{cases}0 & \text { if } t \leq 0, \\ 1-\left(1+t^{1 / \alpha}\right)^{-1} & \text { if } t>0 .\end{cases}
$$

We then set $w(x)=w\left(x_{1}, \ldots, x_{N}\right):=W\left(x_{N}\right)$. Since $1 / \alpha>2$, we have that $w \in$ $C^{2}\left(\mathbb{R}^{N}\right)$, and clearly $\partial_{x_{N}} w \geq 0$.

A straightforward calculation shows that

$$
\Delta_{p} w+f(w)=0,
$$

where

$$
f(r):=\frac{p-1}{\alpha^{p}}(1-r)^{p-1+\alpha p} r^{p-1-\alpha p}(2 r-1+\alpha) \chi_{(0,1)}(r) .
$$

Note that $p-1-\alpha p>1$, thence $f \in C^{1}(\mathbb{R})$.

Moreover,

$$
\left\{\partial_{x_{N}} w=0\right\}=\{w=0\}
$$

and so $f^{\prime}(w(x))=0$ for any $x \in\left\{\partial_{x_{N}} w=0\right\}$. Consequently,

$$
\int_{\partial_{x_{N}} w>0} f^{\prime}(w) \phi^{2} d x=\int_{\mathbb{R}^{N}} f^{\prime}(w) \phi^{2} d x
$$

for any smooth and compactly supported $\phi$.

Accordingly, $w$ is stable, due to (7.1).

The example in Proposition 7.3 shows that case IV in Lemma 4.10 and case D (and, analogously, case C) in Lemma 4.14 may happen.

\section{Proof of Corollary 1.3}

For any $\left(x^{\prime}, x_{3}\right) \in \mathbb{R}^{2} \times \mathbb{R}$ and $r \in \mathbb{R}$, we define

$$
U\left(x^{\prime}, x_{3}\right):= \begin{cases}u\left(x^{\prime}, x_{3}\right) & \text { if } x_{3} \geq 0 \\ -u\left(x^{\prime},-x_{3}\right) & \text { if } x_{3}<0\end{cases}
$$

and

$$
f(r):= \begin{cases}g(r) & \text { if } r \geq 0 \\ -g(-r) & \text { if } r<0\end{cases}
$$


It can be easily seen (see [14] for details) that

$$
\Delta U(x)+f(U(x))=0
$$

for any $x \in \mathbb{R}^{3}$.

Also, by [3, Theorem 1.1], we have that $\partial_{3} u>0$, and so

$$
\partial_{3} U>0 \text {. }
$$

Moreover, $f$ is Lipschitz, since $g(0)=0$. This, (8.1), (8.2) and Theorem 1.2 yield the claim of Corollary 1.3.

\section{Proof of Theorem 1.4}

We take the assumptions of Theorem 1.4.

First, we observe that, without loss of generality, by possibly changing the sign of $u$, we may replace the sign assumption on $f$ by

$$
f \leq 0
$$

\subsection{Area type estimates}

We consider the graph of $u$ as a surface in $\mathbb{R}^{3}$, namely, we set $Y=Y(x):=$ $(x, u(x)) \in \mathbb{R}^{2} \times \mathbb{R}$, that is, we think of $Y$ as a function of $x \in \mathbb{R}^{2}$ in such a way that $Y \in \mathbb{R}^{3}$ belongs to the graph of $u$.

Area estimates are a classical topic in minimal surface theory. Next is a variation of such results in our framework:

Lemma 9.1. There exists a constant $C \geq 1$ in such a way that

$$
\int_{|Y| \leq \rho} a(|\nabla u|)|\nabla u|^{2} d x \leq C \rho^{2}
$$

as long as $\rho \geq C$.

Proof. This proof is inspired by analogous arguments on [24, page 24] and [18, page 403].

To begin, we point out that $\operatorname{ta}(t)>0$ for any $t>0$, due to (1.2), and the map $t \mapsto t a(t)$ is increasing, due to (1.3). As a consequence, from (1.17),

$$
|a(t) t| \leq C
$$

for any $t>0$.

Then, we take $\zeta \in C_{0}^{\infty}\left(B_{2 \rho},[0,1]\right)$ such that $\zeta(x)=1$ if $x \in B_{\rho}, 0 \leq \zeta \leq 1$ and $|\nabla \zeta| \leq \operatorname{const} / \rho$. 
For any $t \in \mathbb{R}$, we also define

$$
\gamma(t):= \begin{cases}1 & \text { if } t \geq \rho, \\ \frac{t+\rho}{2 \rho} & \text { if }|t|<\rho, \\ 0 & \text { if } t \leq-\rho,\end{cases}
$$

and $\varphi(x):=\gamma(u(x)) \zeta(x)$.

Moreover, by (9.2),

$$
\left|\int_{\mathbb{R}^{2}} a(|\nabla u(x)|) \gamma(u(x)) \nabla u(x) \cdot \nabla \zeta(x) d x\right| \leq \text { const } \rho .
$$

Therefore, using $\varphi$ as a test function in (1.1) and recalling (9.1),

$$
\begin{aligned}
& \frac{\text { const }}{\rho} \int_{\substack{|x| \leq \rho \\
|u| \leq \rho}} a(|\nabla u(x)|)|\nabla u|^{2} d x \\
& \leq \int_{\mathbb{R}^{2}} a(|\nabla u(x)|) \gamma^{\prime}(u(x)) \zeta(x)|\nabla u(x)|^{2} d x \\
& =\int_{\mathbb{R}^{2}} f(u(x)) \varphi(x)-a(|\nabla u(x)|) \gamma(u(x)) \nabla u(x) \cdot \nabla \zeta(x) d x \\
& \leq \text { const } \rho,
\end{aligned}
$$

which yields the desired result.

\subsection{Completion of the proof of Theorem 1.4}

We show that the assumptions of Lemma 5.2 are fulfilled. Indeed, here $N=2$, thus (5.4) is not needed; also, (5.3) is implied by (1.18), while (5.1) is warranted by Lemma 9.1.

Then, the use of Lemma 5.2 finishes the proof of Theorem 1.4.

\section{Proof of Theorem 1.5}

We note that Theorem 2.5 holds under the assumptions of Theorem 1.5 (though in this case $\lambda_{1}$ is only non-negative). Accordingly,

$$
\begin{aligned}
& \int_{\mathbb{R}^{2}} \lambda_{2}(|\nabla u(x)|)|\nabla u(x)|^{2} \kappa^{2}(x) \varphi^{2}(x) d x \\
& \leq \int_{\mathbb{R}^{2}}|\nabla u(x)|^{2}(A(\nabla u(x)) \nabla \varphi(x)) \cdot \nabla \varphi(x) d x
\end{aligned}
$$


for any locally Lipschitz, compactly supported function $\varphi$, where $\kappa(x)$ is the curvature of the level set passing through $x$.

Then, by (A1),

$$
\int_{\mathbb{R}^{2}} \lambda_{2}(|\nabla u(x)|)|\nabla u(x)|^{2} \kappa^{2}(x) \varphi^{2}(x) d x \leq K \int_{\mathbb{R}^{2}}|\nabla \varphi(x)|^{2} d x .
$$

As a consequence, arguing as in Corollary 2.6, since $\nabla u$ never vanishes, we conclude that the level sets are regular curves with vanishing curvatures, thence straight lines.

\section{References}

[1] G. Alberti, L. Ambrosio and X. CABré, On a long-standing conjecture of E. De Giorgi: symmetry in $3 D$ for general nonlinearities and a local minimality property, Acta Appl. Math. 65 (2001), 9-33.

[2] L. Ambrosio and X. CABRÉ, Entire solutions of semilinear elliptic equations in $\mathbb{R}^{3}$ and a conjecture of De Giorgi, J. Amer. Math. Soc. 13 (2000) (electronic), 725-739.

[3] H. Berestycki, L. CAFFARElli and L. Nirenberg, Further qualitative properties for elliptic equations in unbounded domains, Ann. Scuola Norm. Sup. Pisa Cl. Sci. 25 (19971998), 69-94.

[4] S. BERNSTEIN, Über ein geometrisches Theorem und seine Anwendung auf die partiellen Differentialgleichungen vom elliptischen Typus, Math. Z. 26 (1927), 551-558.

[5] L. CAFFARElli, N. Garofalo and F. SEGÀla, A gradient bound for entire solutions of quasi-linear equations and its consequences, Comm. Pure Appl. Math. 47 (1994), 14571473.

[6] L. DAMASCELli and B. SCIUNZI, Regularity, monotonicity and symmetry of positive solutions of m-Laplace equations, J. Differential Equations 206 (2004), 483-515.

[7] D. Danielli and N. Garofalo, Properties of entire solutions of non-uniformly elliptic equations arising in geometry and in phase transitions, Calc. Var. Partial Differential Equations 15 (2002), 451-491.

[8] E. DE GIORGI, Convergence problems for functionals and operators, In: "Proceedings of the International Meeting on Recent Methods in Nonlinear Analysis (Rome, 1978)", Bologna, 1979, 131-188, Pitagora Editrice.

[9] E. DiBenedetto, $C^{1+\alpha}$ local regularity of weak solutions of degenerate elliptic equations, Nonlinear Anal. 7 (1983), 827-850.

[10] L. C. Evans and R. F. GARIEPY, "Measure theory and fine properties of functions", Studies in Advanced Mathematics. CRC Press, Boca Raton, FL, 1992.

[11] A. FARINA, "Propriétés qualitatives de solutions d'équations et systèmes d'équations nonlinéaires", Habilitation à diriger des recherches, Paris VI, 2002.

[12] A. FARINA, One-dimensional symmetry for solutions of quasilinear equations in $\mathbb{R}^{2}$, Boll. Unione Mat. Ital. Sez. B Artic. Ric. Mat. 6 (2003), 685-692.

[13] A. FARINA, Liouville-type theorems for elliptic problems, In: "Handbook of Differential Equations: Stationary Partial Differential Equations" M. Chipot (ed.), Vol. IV Elsevier B. V., Amsterdam, 2007, 61-116.

[14] A. FARINA, B. ScIUnZI and E. VAldinoci, Bernstein and De Giorgi type problems: new results via a geometric approach, Preliminary version of this paper, available at http://cvgmt.sns.it/papers/, 2007.

[15] A. FARINA and E. VALDINOCI, $1 D$ symmetry for solutions of semilinear and quasilinear elliptic equations, Preprint, 2008. 
[16] D. FISCHER-COLBRIE and R. SCHOEN, The structure of complete stable minimal surfaces in 3-manifolds of nonnegative scalar curvature, Comm. Pure Appl. Math. 33 (1980), 199211.

[17] N. Ghoussoub and C. GuI, On a conjecture of De Giorgi and some related problems, Math. Ann. 311 (1998), 481-491.

[18] D. Gilbarg and N. S. Trudinger, "Elliptic partial differential equations of second order", Classics in Mathematics, Springer-Verlag, Berlin, 2001.

[19] E. H. LIEB and M. Loss, "Analysis", Vol. 14 of Graduate Studies in Mathematics, American Mathematical Society, Providence, RI, 1997.

[20] L. ModicA, A gradient bound and a Liouville theorem for nonlinear Poisson equations, Comm. Pure Appl. Math. 38 (1985), 679-684.

[21] W. F. Moss and J. PiePEnBRInK, Positive solutions of elliptic equations, Pacific J. Math. 75 (1978), 219-226.

[22] O. SAVIN, Regularity of flat level sets in phase transitions, to appear in Ann. of Math. 2008.

[23] E. SERnESI, "Geometria 2", Bollati Boringhieri, Torino, 1994.

[24] L. Simon, "Singular Sets and Asymptotics in Geometric Analysis", Lipschitz Lectures. Institut für Angewandte Mathematik, http://math.stanford.edu/ lms/lipschitz/lipschitz.pdf, Bonn, 2007.

[25] Y. S. SiRE and E. VAlDinOCI, Fractional Laplacian phase transitions and boundary reactions: a geometric inequality and a symmetry result, preprint, 2008.

[26] P. Sternberg and K. Zumbrun, Connectivity of phase boundaries in strictly convex domains, Arch. Ration. Mech. Anal. 141 (1998), 375-400.

[27] P. Sternberg and K. Zumbrun, A Poincaré inequality with applications to volumeconstrained area-minimizing surfaces, J. Reine Angew. Math. 503 (1998), 63-85.

[28] P. TOLKSDORF, Regularity for a more general class of quasilinear elliptic equations, J. Differential Equations 51 (1984), 126-150.

[29] K. UhlenbeCK, Regularity for a class of non-linear elliptic systems, Acta Math. 138 (1977), 219-240.

[30] E. VAlDinOCI, B. ScIUNZI and V. O. SAVIN, Flat level set regularity of p-Laplace phase transitions, Mem. Amer. Math. Soc. 182 (2006), vi-144.

LAMFA - CNRS UMR 6140

Université de Picardie Jules Verne

Faculté de Mathématiques et d'Informatique 33, rue Saint-Leu

80039 Amiens CEDEX 1, France

alberto.farina@u-picardie.fr

Università di Roma Tor Vergata

Dipartimento di Matematica

Via della ricerca scientifica, 1

00133 Roma, Italy

sciunzi@mat.uniroma2.it

valdinoci@mat.uniroma2.it 